\title{
CO emission in distant galaxies on and above the main sequence ${ }^{\star}$
}

\author{
F. Valentino ${ }^{1,2}$, E. Daddi ${ }^{3}$, A. Puglisi ${ }^{3,4}$, G. E. Magdis ${ }^{1,2,5,6}$, D. Liu ${ }^{7}$, V. Kokorev ${ }^{1,2}$, I. Cortzen ${ }^{1,2}$, S. Madden ${ }^{3}$, \\ M. Aravena ${ }^{8}$, C. Gómez-Guijarro ${ }^{3}$, M.-Y. Lee ${ }^{9}$, E. Le Floc'h' ${ }^{3}$, Y. Gao ${ }^{10,11}$, R. Gobat ${ }^{12}$, F. Bournaud ${ }^{3}$, \\ H. Dannerbauer ${ }^{13,14}$, S. Jin ${ }^{13,14}$, M. E. Dickinson ${ }^{15}$, J. Kartaltepe ${ }^{16}$, and D. Sanders ${ }^{17}$ \\ 1 Cosmic Dawn Center (DAWN), Copenhagen, Denmark \\ 2 Niels Bohr Institute, University of Copenhagen, Lyngbyvej 2, 2100 Copenhagen Ø, Denmark \\ e-mail: francesco.valentino@nbi.ku.dk \\ 3 AIM, CEA, CNRS, Université Paris-Saclay, Université Paris Diderot, Sorbonne Paris Cité, 91191 Gif-sur-Yvette, France \\ ${ }^{4}$ Center for Extragalactic Astronomy, Durham University, South Road, Durham DH13LE, UK \\ 5 DTU-Space, Technical University of Denmark, Elektrovej 327, 2800 Kgs. Lyngby, Denmark \\ ${ }^{6}$ Institute for Astronomy, Astrophysics, Space Applications and Remote Sensing, National Observatory of Athens, 15236 Athens, \\ Greece \\ 7 Max Planck Institute for Astronomy, Königstuhl 17, 69117 Heidelberg, Germany \\ 8 Núcleo de Astronomía, Facultad de Ingeniería y Ciencias, Universidad Diego Portales, Av. Ejército 441, Santiago, Chile \\ 9 Korea Astronomy and Space Science Institute, 776 Daedeokdae-ro, 34055 Daejeon, Republic of Korea \\ 10 Purple Mountain Observatory \& Key Laboratory for Radio Astronomy, Chinese Academy of Sciences, 10 Yuanhua Road, Nanjing \\ 210033, PR China \\ 11 Department of Astronomy, Xiamen University, Xiamen, Fujian 361005, PR China \\ 12 Instituto de Física, Pontificia Universidad Católica de Valparaíso, Casilla 4059, Valparaíso, Chile \\ 13 Instituto de Astrofísica de Canarias (IAC), 38205 La Laguna, Tenerife, Spain \\ 14 Universidad de La Laguna, Dpto. Astrofísica, 38206 La Laguna, Tenerife, Spain \\ 15 NSF's National Optical-Infrared Astronomy Research Laboratory, 950 North Cherry Avenue, Tucson, AZ 85719, USA \\ 16 School of Physics and Astronomy, Rochester Institute of Technology, 84 Lomb Memorial Drive, Rochester, NY 14623, USA \\ 17 University of Hawaii, Institute for Astronomy, 2680 Woodlawn Drive, Honolulu, HI 96822, USA
}

Received 1 May 2020 / Accepted 21 June 2020

\begin{abstract}
We present the detection of multiple carbon monoxide CO line transitions with ALMA in a few tens of infrared-selected galaxies on and above the main sequence at $z=1.1-1.7$. We reliably detected the emission of $\mathrm{CO}(5-4), \mathrm{CO}(2-1)$, and $\mathrm{CO}(7-6)+[\mathrm{C} \mathrm{I}]\left({ }^{3} P_{2}-\right.$ $\left.{ }^{3} P_{1}\right)$ in 50, 33, and 13 galaxies, respectively, and we complemented this information with available $\mathrm{CO}(4-3)$ and $[\mathrm{C} \mathrm{I}]\left({ }^{3} P_{1}-{ }^{3} P_{0}\right)$ fluxes for part of the sample, and by modeling of the optical-to-millimeter spectral energy distribution. We retrieve a quasi-linear relation between $L_{\mathrm{IR}}$ and $\mathrm{CO}(5-4)$ or $\mathrm{CO}(7-6)$ for main-sequence galaxies and starbursts, corroborating the hypothesis that these transitions can be used as star formation rate (SFR) tracers. We find the CO excitation to steadily increase as a function of the star formation efficiency, the mean intensity of the radiation field warming the dust $(\langle U\rangle)$, the surface density of SFR $\left(\Sigma_{\mathrm{SFR}}\right)$, and, less distinctly, with the distance from the main sequence $(\Delta \mathrm{MS})$. This adds to the tentative evidence for higher excitation of the $\mathrm{CO}+[\mathrm{CI}]$ spectral line energy distribution (SLED) of starburst galaxies relative to that for main-sequence objects, where the dust opacities play a minor role in shaping the high- $J$ CO transitions in our sample. However, the distinction between the average SLED of upper main-sequence and starburst galaxies is blurred, driven by a wide variety of intrinsic shapes. Large velocity gradient radiative transfer modeling demonstrates the existence of a highly excited component that elevates the CO SLED of high-redshift main-sequence and starbursting galaxies above the typical values observed in the disk of the Milky Way. This excited component is dense and it encloses $\sim 50 \%$ of the total molecular gas mass in main-sequence objects. We interpret the observed trends involving the CO excitation as to be mainly determined by a combination of large SFRs and compact sizes, as a large $\Sigma_{\text {SFR }}$ is naturally connected with enhanced dense molecular gas fractions and higher dust and gas temperatures, due to increasing ultraviolet radiation fields, cosmic ray rates, as well as dust and gas coupling. We release the full data compilation and the ancillary information to the community.
\end{abstract}

Key words. galaxies: evolution - galaxies: ISM - galaxies: starburst - galaxies: high-redshift

\section{Introduction}

Since its first detection in external galaxies a few decades ago, the prominent role of the molecular gas in determining the evolution of galaxies has been established by constantly growing

\footnotetext{
* The data compilation described in Table D.1 is only available at the CDS via anonymous ftp to cdsarc.u-strasbg. fr (130.79.128.5) or via http://cdsarc.u-strasbg.fr/viz-bin/cat/J/A+A/641/ A155
}

evidence and interpreted by progressively more sophisticated theoretical arguments (e.g., Young \& Scoville 1991; Solomon \& Vanden Bout 2005; Carilli \& Walter 2013; Hodge \& da Cunha 2020 , for reviews). On the one hand, the detection of tens of different molecular transitions in local molecular clouds and resolved nearby galaxies, spanning a wide range of properties, allowed for a detailed description of the processes regulating the physics of the interstellar medium (ISM). On the other hand, the observation of a handful of species and lines in unresolved 
galaxies at various redshifts has been instrumental in identifying the main transformations that galaxy populations undergo with time. In particular, it is now clear that the majority of galaxies follows a series of scaling relations connecting their star formation rates (SFRs), the available molecular and atomic gas reservoirs $\left(M_{\mathrm{gas}}, M_{\mathrm{HI}}\right)$ and their densities and temperatures, the stellar and dust masses $\left(M_{\star}, M_{\text {dust }}\right)$, metallicities $(Z)$, sizes, and several other properties derived from the combination of these parameters. Two relations have received special attention in the past decade: the so-called main sequence (MS) of star-forming galaxies, a quasi-linear and relatively tight $(\sigma \sim 0.3$ dex $)$ correlation between $M_{\star}$ and SFR (Brinchmann et al. 2004; Noeske et al. 2007; Daddi et al. 2007; Elbaz et al. 2007; Rodighiero et al. 2011; Whitaker et al. 2012; Speagle et al. 2014; Sargent et al. 2014; Schreiber et al. 2015) and the Schmidt-Kennicutt (SK) relation between the surface densities of SFR and gas mass $\left(\Sigma_{\text {SFR }}-\Sigma_{\text {gas }}\right.$, Schmidt 1959; Kennicutt 1998). Only a minor fraction of massive star-forming galaxies, dubbed starbursts (SBs), deviate from the MS, displaying exceptional SFRs for their $M_{\star}$ (Rodighiero et al. 2011), and potentially larger $\Sigma_{\mathrm{SFR}}$ at fixed $\Sigma_{\text {gas }}$ (Daddi et al. 2010a; Sargent et al. 2014; Casey et al. 2014). These objects are generally related to recent merger events, at least in the local Universe, and they can be easily spotted as bright beacons in the far-infrared and (sub)millimeter regimes, owing to their strong dust emission exceeding $L_{\mathrm{IR}}>10^{11-12} L_{\odot}$ ((Ultra)Luminous InfraRed Galaxies, (U)LIRGs, Sanders \& Mirabel 1996).

It has also become evident that the normalization of the MS rapidly increases with redshift: distant galaxies form stars at higher paces than in the local Universe, at fixed stellar mass (e.g., Daddi et al. 2007; Elbaz et al. 2007; Whitaker et al. 2012; Speagle et al. 2014; Schreiber et al. 2015). This trend could be explained by the availability of copious molecular gas at high redshift (Daddi et al. 2010a; Tacconi et al. 2010, 2018; Scoville et al. 2017a; Riechers et al. 2019; Decarli et al. 2019; Liu et al. 2019a), ultimately regulated by the larger accretion rates from the cosmic web (Kereš et al. 2005; Dekel et al. 2009a). Moreover, higher SFRs could be induced by an increased efficiency of star formation due to the enhanced fragmentation in gas-rich, turbulent, and gravitationally unstable high-redshift disks (Bournaud et al. 2007, 2010; Dekel et al. 2009b; Ceverino et al. 2010; Dekel \& Burkert 2014), reflected on their clumpy morphologies (Elmegreen et al. 2007; Förster Schreiber et al. 2011; Genzel et al. 2011; Guo et al. 2012, 2015; Zanella et al. 2019). IR-bright galaxies with prodigious SFRs well above the level of the MS are observed also in the distant Universe, but their main physical driver is a matter of debate. While a star formation efficiency $\left(\mathrm{SFE}=\mathrm{SFR} / M_{\text {gas }}\right.$ ) monotonically increasing with the distance from the main sequence $(\triangle \mathrm{MS}=$ SFR/SFR ${ }_{M S}$, Genzel et al. 2010, 2015; Magdis et al. 2012; Tacconi et al. 2018, 2020) could naturally explain the existence of these outliers, recent works suggest the concomitant increase of gas masses as the main driver of the starbursting events (Scoville et al. 2016; Elbaz et al. 2018). In addition, if many bright starbursting (sub)millimeter galaxies (SMGs, Smail et al. 1997) are indeed merging systems as in the local Universe (Gómez-Guijarro et al. 2018, and references therein), there are several well documented cases of SMGs hosting orderly rotating disks at high redshift (e.g., Hodge et al. 2016, 2019; Drew et al. 2020), disputing the pure merger scenario. The same definition of starbursts as galaxies deviating from the main sequence has been recently questioned with the advent of high spatial resolution measurements of their dust and gas emission. Compact galaxies with short depletion timescales typical of SBs are now routinely found on the MS, being possibly on their way to leave the sequence (Barro et al. 2017a; Popping et al. 2017; Elbaz et al. 2018; Gómez-Guijarro et al. 2019; Puglisi et al. 2019; JiménezAndrade et al. 2019); or galaxies moving within the MS scatter, due to mergers unable to efficiently boost the star formation (Fensch et al. 2017) or owing to gravitational instabilities and gas radial redistribution (Tacchella et al. 2016).

In this framework, a primary source of confusion stems from the relatively limited amount of information available for sizable samples of high-redshift galaxies, homogeneously selected on and above the main sequence. While a fine sampling of the far-IR spectral energy distribution (SED) has now become more accessible and a fundamental source to derive properties as the dust mass, temperature, and luminosity (e.g., Simpson et al. 2014, 2020; Scoville et al. 2016; Dunlop et al. 2017; Brisbin et al. 2017; Strandet et al. 2017; Franco et al. 2018; Zavala et al. 2018; Liu et al. 2019b; Dudzevičiūtè et al. 2020; Hodge \& da Cunha 2020, to mention a few recent high-resolution surveys in the (sub)millimeter), direct spectroscopic measurements of the cold gas in distant galaxies remain remarkably time consuming. As a result, systematic investigations of the gas properties focused on either one line transition in large samples of galaxies (e.g., Le Fèvre et al. 2020; Freundlich et al. 2019; Tacconi et al. 2018, 2020), or several lines in sparser samples, often biased toward the brightest objects as (lensed) SMGs or quasars (e.g., Carilli \& Walter 2013; Bothwell et al. 2013; Spilker et al. 2014; Yang et al. 2017; Cañameras et al. 2018; Dannerbauer et al. 2019). Moreover, the spectroscopic study of normal MS galaxies at high redshift has been primarily devoted to the determination of the total molecular gas masses and fractions via the follow-up of low- $J$ carbon monoxide transitions (CO $(1-0)$ to CO (3 - 2), Dannerbauer et al. 2009; Daddi et al. 2010a; Tacconi et al. 2010, 2020; Freundlich et al. 2019), with a few exceptions ([C I], Valentino et al. 2018, 2020; Bourne et al. 2019; Brisbin et al. 2019; [C II], Capak et al. 2015; Zanella et al. 2018; Le Fèvre et al. 2020). Little is known about the denser and warmer phases in distant normal disks, but these components might hold the key to reach a deeper understanding of the galaxy growth, being naturally associated with the star-forming gas.

A few pilot studies specifically targeting mid- $J$ CO transitions in MS galaxies suggest the existence of significant pockets of such dense and warm molecular gas up to $z \sim 3$ (Daddi et al. 2015; Brisbin et al. 2019; Cassata et al. 2020), along with more routinely detected large cold reservoirs traced by low- $J$ lines (Dannerbauer et al. 2009; Aravena et al. 2010, 2014). The observed CO line luminosities of moderately excited transitions as $\mathrm{CO}(5-4)$ further correlate almost linearly with the total IR luminosity $L_{\mathrm{IR}}$ (Daddi et al. 2015), similarly to what is observed for local IR bright objects and distant SMGs (Greve et al. 2014; Liu et al. 2015a; Lu et al. 2015, 2017; Kamenetzky et al. 2016), suggesting their potential use as SFR tracers. Moreover, these studies show evidence of CO spectral line energy distributions (SLEDs) significantly more excited in MS galaxies than what observed in the Milky Way, but less than local (U)LIRGs and high-redshift SMGs (Dannerbauer et al. 2009; Daddi et al. 2015; Cassata et al. 2020). While not necessarily good proxies of the mode of star formation (secular vs bursty) per se, (CO) SLEDs are relevant if they can constrain the fraction of dense molecular gas (Daddi et al. 2015), and they remain a precious source of information on the processes heating and exciting the ISM. This has been extensively proven by detailed studies of local galaxies, including spirals, ongoing mergers, starbursts, and active nuclear 
regions (Panuzzo et al. 2010; Papadopoulos et al. 2010a,b, 2012; Rangwala et al. 2011; Kamenetzky et al. 2012, 2017; Schirm et al. 2014; Lu et al. 2014, 2017; Wu et al. 2015; Mashian et al. 2015; Rosenberg et al. 2015; Kamenetzky et al. 2016; Lee et al. 2019, among the others). However, the study of warm and dense molecular gas in distant MS galaxies remains limited to a handful of objects to date.

Here we present the first results of a new multicycle campaign with the Atacama Large Millimeter Array (ALMA), whose impressive capabilities allowed for the survey of several species in the span of a few minutes of on-source integration. We targeted multiple $\mathrm{CO}(\mathrm{CO}(2-1), \mathrm{CO}(4-3), \mathrm{CO}(5-4), \mathrm{CO}(7-$ 6)) and neutral atomic carbon $\left([\mathrm{C} \mathrm{I}]\left({ }^{3} P_{1}-{ }^{3} P_{0}\right),[\mathrm{C} \mathrm{I}]\left({ }^{3} P_{2}-{ }^{3} P_{1}\right)\right)$ line emissions in a sample of a few tens of main-sequence and starburst galaxies at $z \sim 1.3$. Our main goal is to explore the excitation conditions of the molecular gas in normal disks and bursty objects and to relate it with their star formation modes, in the attempt to cast new light on the formation scenarios mentioned above. In particular, we aim to explore that portion of the parameter space spanned by mid- and high- $J$ CO transitions in distant normal main-sequence galaxies currently lacking a systematic coverage. While admittedly not comparable with the wealth of information available for local objects and on subgalactic scales, the combination of new ALMA data and archival work is a first step toward the multiline and large statistical studies necessary to fully unveil the origin of the trends for the normal MS systems discussed above.

Part of the data has been already used in previous works. In particular, Puglisi et al. (2019) focused on the far-IR sizes of our sample and anticipated the blurred difference between upper MS and SB galaxies mentioned above, revealing a significant population of post-starburst galaxies on the main sequence. A more articulated analysis of the role of compactness on galaxy evolution is in preparation (Puglisi et al., in prep.). We have also discussed the neutral carbon emission in two articles (Valentino et al. 2018, 2020, V18 and V20 in the rest of this work). Here we present the details of the observational campaign, the target selection, the data reduction and analysis, and we release all the measurements to the community. The present release supersedes the previous ones and should be taken as reference. We then explore and interpret the basic correlations among several observables and the properties that they are connected with. We further investigate the excitation conditions of MS and SB galaxies by presenting the observed high/low- $J$ CO line ratios as a function of the fundamental properties of the sample; by attempting a simple modeling of the CO SLEDs; and by comparing the latter with state-of-the-art simulations and analytical predictions.

In the main body of the manuscript we present the primary scientific results and we provide the essential technical elements. We refer the reader interested in finer details to the appendices. Unless stated otherwise, we assume a $\Lambda$ CDM cosmology with $\Omega_{\mathrm{m}}=0.3, \Omega_{\Lambda}=0.7$, and $H_{0}=70 \mathrm{~km} \mathrm{~s}^{-1} \mathrm{Mpc}^{-1}$ and a Chabrier initial mass function (IMF, Chabrier 2003). All magnitudes are expressed in the $\mathrm{AB}$ system. All the literature data have been homogenized with our conventions.

\section{Survey description}

\subsection{The primary $\mathrm{CO}(5-4)$ sample}

The survey was originally designed to observe CO $(5-4)$ in a statistical sample of field IR-bright galaxies, distributed on and above the main sequence at $z \simeq 1.1-1.7$ in the COSMOS area (Scoville et al. 2007). The program was prepared

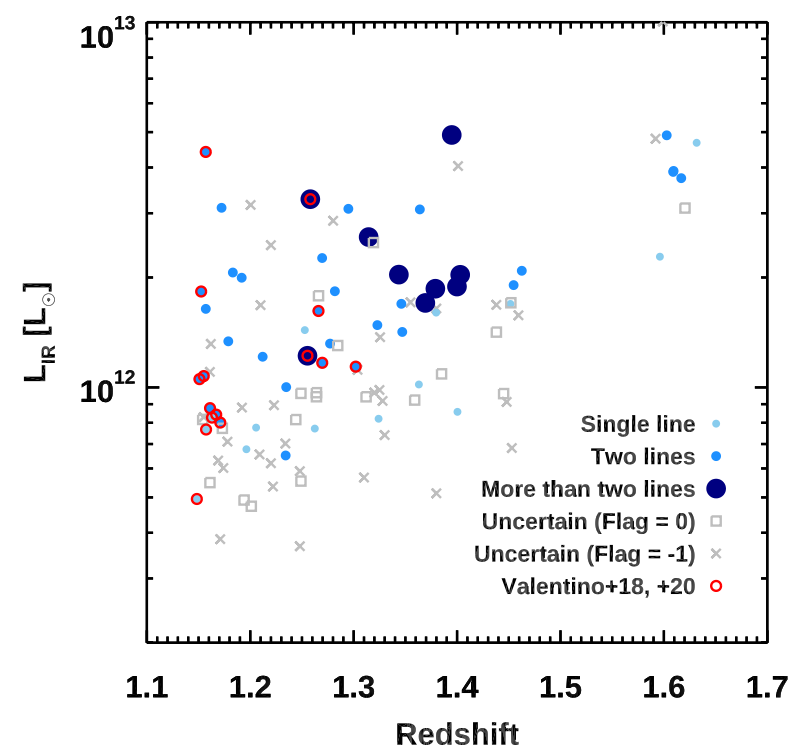

Fig. 1. Survey design: the redshifts and total IR luminosities distributions of the primary $\mathrm{CO}(5-4)$ sample of our campaign. Gray crosses mark uncertain sources due to poor optical redshifts (Flag $=-1$ ). Gray open squares indicate uncertain upper limits on every covered line with trustable $z_{\text {spec,opt }}($ Flag $=0)$. Light blue, blue, and navy blue filled circles indicate reliable sources with usable information about one, two, or more than two transitions from this work (Flag $\geq 0.5$ ). Red open circles mark the objects already presented in V18 and V20. Galaxies without an updated far-IR modeling based on the photometry in Jin et al. (2018) are not shown. The flagging classification and the definition of reliable and uncertain data are described in Sect. 3.2.2.

for ALMA Cycle 3. The targets had available stellar mass estimates (Muzzin et al. 2013; Laigle et al. 2016), a spectroscopic confirmation from near-IR and optical observations from the COSMOS master catalog (Salvato et al., in prep.), and a Herschel/PACS $100 \mu \mathrm{m}$ or $160 \mu \mathrm{m} 3 \sigma$ detection in the PEP catalog (Lutz et al. 2011). Initially we considered 178 objects with a predicted $\mathrm{CO}(5-4)$ line flux of $I_{54}>1 \mathrm{Jy} \mathrm{km} \mathrm{s}^{-1}$ over $400 \mathrm{~km} \mathrm{~s}^{-1}$, based on the IR luminosity and the $L_{\mathrm{IR}}-L_{\mathrm{CO}(5-4)}^{\prime}$ relation from Daddi et al. (2015, D15 hereafter). This constant flux cut corresponds to $L_{\mathrm{IR}} \gtrsim 10^{12} L_{\odot}$ in the redshift interval under consideration. We then grouped these objects in frequency ranges within ALMA Band 6, allowing for potential individual detections in less than two minutes of on-source integration, while minimizing the overheads. The final spectral sampling includes 123 primary targets homogeneously spread over the $z-L_{\mathrm{IR}}$ space (Fig. 1), with mean and median stellar masses of $M_{\star}=10^{10.7} M_{\odot}$ and a 0.4 dex dispersion. We refer to these galaxies as the "primary sample" of our survey. Since a new "super-deblended" IR catalog for the COSMOS field became available (Jin et al. 2018), a posteriori we remodeled the SED of each object and updated the initial estimates of $L_{\mathrm{IR}}$ (Sect. 3.3). Twelve targets from the PEP catalog do not have a deblended counterpart in Jin et al. (2018), and, thus, do not have an updated estimate of $L_{\mathrm{IR}}$. Moreover, two objects significantly detected in the IR do not have a certain optical counterpart and, thus, a stellar mass estimate. Excluding these sources, based on the new modeling 78 targets lie on the main sequence as parameterized by Sargent et al. (2014) and 31 are classified as starbursts $(\geq 3.5 \times$ above the main sequence). The threshold for the definition of starburst is arbitrary and set in order to be consistent with V18 and V20. Five objects have a dominant dusty torus component in the IR SED (see Sect. 3.3). The final distribution of the targets in 
Table 1. Summary of the multicycle ALMA campaign presented in this work.

\begin{tabular}{ccccccc}
\hline \hline Epoch & Band & Transitions & Sample & $\begin{array}{c}\left\langle t_{\text {int }}\right\rangle^{(a)} \\
{[\mathrm{min}]}\end{array}$ & Beam size & $\begin{array}{c}\langle\mathrm{rms}\rangle \\
{[\mathrm{mJy}]}\end{array}$ \\
\hline Cycle 3 & Band 6 & $\mathrm{CO}(5-4)$ & 123 & 1.6 & $0.8^{\prime \prime}$ & 0.5 \\
Cycle 4 & Band 3 & $\mathrm{CO}(2-1)$ & 75 & 4 & $1.4^{\prime \prime}$ & 0.375 \\
Cycle 7 & Band 7 & $\mathrm{CO}(7-6),[\mathrm{CI}]\left({ }^{3} P_{2}-{ }^{3} P_{1}\right)$ & 15 & 24 & $1.0^{\prime \prime}$ & $0.25^{(b)}$ \\
\hline
\end{tabular}

Notes. ${ }^{(a)}$ Average on-source integration time. ${ }^{(b)}$ Average rms.

the $z-L_{\mathrm{IR}}$ space with the updated IR modeling is shown in Fig. 1 We note that the initial requirement of a PACS detection steered the sample toward upper main-sequence objects and warm dust temperatures $T_{\text {dust }} \gtrsim 30 \mathrm{~K}$.

\subsection{The $\mathrm{CO}(2-1)$ and $\mathrm{CO}(7-6)$ follow-up}

The follow-up of the $\mathrm{CO}(2-1)$ transition during ALMA Cycle 4 was focused on a subsample of $75 / 123$ objects above a constant line flux threshold of $I_{21} \geq 0.75 \mathrm{Jy} \mathrm{km} \mathrm{s}^{-1}$ over $400 \mathrm{~km} \mathrm{~s}^{-1}$. We gathered the potential targets in blocks of frequency settings to contain the overheads, shrinking the initial pool of galaxies with $\mathrm{CO}(5-4)$ coverage. Similar considerations apply for the most recent ALMA program in Cycle 7 , targeting [C I $]\left({ }^{3} P_{2}-\right.$ $\left.{ }^{3} P_{1}\right)+\mathrm{CO}(7-6)$ in 15 galaxies with potential simultaneous visibility of $[\mathrm{CI}]\left({ }^{3} P_{1}-{ }^{3} P_{0}\right)$. In this case, we sacrificed the flux completeness down to a constant threshold to obtain the largest number of multiline measurements, adjusting the detection limit of every block of observations to the dimmest source in each pool.

Finally, 15/123 galaxies have at least a detection of [C I $]\left({ }^{3} P_{1}-{ }^{3} P_{0}\right), \mathrm{CO}(4-3),[\mathrm{C} \mathrm{I}]\left({ }^{3} P_{2}-{ }^{3} P_{1}\right)$, and $\mathrm{CO}(7-6)$ from V18 and V20. In the latter, we operated the target selection following a similar strategy as the one outlined here, namely by imposing comparable $L_{\mathrm{IR}}$ and redshift cuts. Figure 1 shows the combined information on every targeted line available for the overall sample studied here. We return to the details of the detection rate below.

\section{Data and analysis}

\subsection{ALMA Observations}

The primary sample of 123 targets described in Sect. 2 was observed in Band 6 during ALMA Cycle 3 (\#2015.1.00260.S, PI: E. Daddi). The goal of a flux density rms of $0.5 \mathrm{mJy}$, necessary to detect a line flux of $I_{54}>1 \mathrm{Jy} \mathrm{km} \mathrm{s}^{-1}$ over $400 \mathrm{~km} \mathrm{~s}^{-1}$ at $>5 \sigma$, was reached in $\lesssim 1.7 \mathrm{~min}$ of integration on source per target (Table 1). The whole program was completed, delivering cubes for all targets with an average beam size of $\sim 0.8^{\prime \prime}$. The subsample of $75 / 123$ sources for the $\operatorname{CO}(2-1)$ follow-up was observed in Band 3 during ALMA Cycle 4 (\#2016.1.00171.S, PI: E. Daddi). With an average on-source integration of $\sim 4 \mathrm{~min}$, the observations matched the requested rms of $0.375 \mathrm{mJy}$, allowing us to detect $I_{21}>0.75 \mathrm{Jy} \mathrm{km} \mathrm{s}^{-1}$ over $400 \mathrm{~km} \mathrm{~s}^{-1}$ at $>5 \sigma$, in principle. Again, the full program was observed and delivered, providing cubes with an average beam size of $\sim 1.4^{\prime \prime}$. Finally, 15/123 galaxies were observed in Band 7 to detect $[\mathrm{CI}]\left({ }^{3} P_{2}-{ }^{3} P_{1}\right)+\mathrm{CO}(7-6)$ during Cycle 7 (\#2019.1.01702.S, PI: F. Valentino), reaching a flux density rms of $0.190-0.315 \mathrm{mJy}$. We underline that the idea behind this program was to maximize the number of submillimeter line detections per source, rather than reaching a constant flux depth for the whole sample. Thus, the limiting rms of every block of observations was adapted to the faintest object in each group. The final average beam size is $\sim 1.0^{\prime \prime}$.

As mentioned in Sect. 2, 15/123 galaxies in the primary sample have one or multiple detections of the neutral atomic carbon $[\mathrm{CI}]$ transitions, $\mathrm{CO}(4-3)$, and $\mathrm{CO}(7-6)$, as a result of an independent campaign carried out during ALMA Cycle 4 and 6 (\#2016.1.01040.S and \#2018.1.00635.S, PI: F. Valentino). The delivered Band 6 data have an average beam size of $2.0 \times 11^{\prime \prime} 7$, reaching a flux threshold of $\sim 0.15 \mathrm{Jy} \mathrm{km} \mathrm{s}^{-1}$ per beam for a line width of $400 \mathrm{~km} \mathrm{~s}^{-1}$ (V18). The follow-up in Band 7 reached and rms of $0.064-0.58 \mathrm{mJy}$, and the resulting cubes have an average beam size of $\sim 0.9^{\prime \prime}$. We refer the reader to V18 and V20 for more details.

\subsection{Data reduction and spectral extraction}

We reduced the data following the iterative procedure described in D15 (see also V18, V20, Coogan et al. 2018; Puglisi et al. 2019; Jin et al. 2019 for reference). We calibrated the cubes using the standard pipeline with CASA (McMullin et al. 2007) and analyzed them with customized scripts within GILDAS ${ }^{1}$ (Guilloteau et al. 2000). For each source, we combined all the available ALMA observations in the $u v$ space allowing for an arbitrary renormalization of the signal for all tracers. We then modeled each source as circular Gaussian in the $u v$ plane to extract the spectrum, allowing the source position, size, and total flux per channel to vary. Finally, the spectrum was obtained from the fit total fluxes per channel. This method has no obvious bias against fitting in the image plane (Coogan et al. 2018), but it has more flexibility in fitting parameters. We iteratively looked for spatially extended signal from the $\mathrm{S} / \mathrm{N}$-weighted combination of all the available tracers, resorting to a point source extraction whenever this search was not successful (D15). In the former case, we could safely measure the size of the emitting source and recover the total flux. When using a point source extraction, we derived upper limits on the sizes and estimated the flux losses as detailed in Appendix A. Finally, we estimated the probability of each galaxy to be unresolved $\left(P_{\text {unres }}\right)$ by comparing the $\chi^{2}$ of the best-fit circular Gaussian and the point source extraction (Puglisi et al. 2019). Using a combination of low- and high-J CO transitions, [C I] lines, and continuum emission, the sizes should be considered as representative of the extension of the molecular gas and cold dust in our galaxies. However, we note that the size is primarily driven by $\mathrm{CO}(5-4)$ and its underlying dust continuum emission (see Fig. 1 of Puglisi et al. 2019).

\subsubsection{Line and continuum flux measurements}

We blindly scanned the extracted spectra looking for potential line emission. We did so by looking for the maximum $\mathrm{S} / \mathrm{N}$

1 http://wWw.iram.fr/IRAMFR/GILDAS 

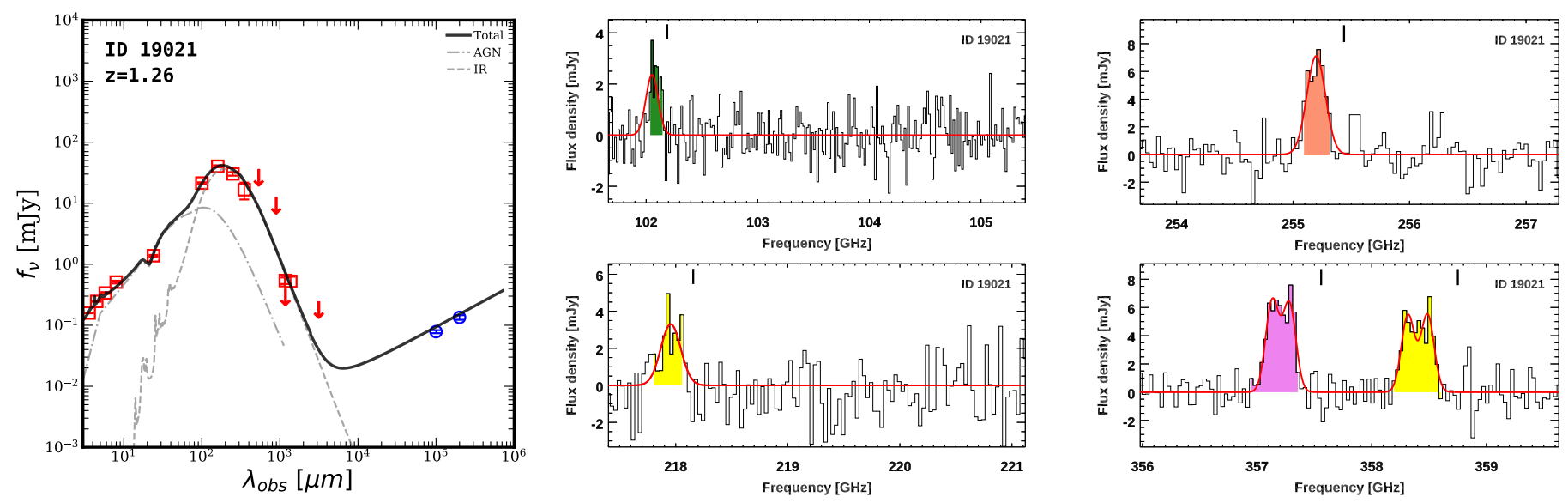

Fig. 2. Example of ALMA spectra and far-IR spectral energy distribution. Left: red open squares and arrows indicate detections and $3 \sigma$ upper limits on the IR photometry that we modeled. Blue open circles indicate radio measurements, which we did not include in the fitting. The gray dashed and dotted-dashed lines mark the best-fit Draine \& Li (2007) and Mullaney et al. (2011) templates for the star-forming and AGN components, respectively. A stellar template was included when modeling the IRAC bands, but we do not show it to avoid confusion. The black solid line indicates the sum of all the templates. Right: ALMA spectra with detected $\mathrm{CO}(2-1)$ (green), $\mathrm{CO}(5-4)$ (orange), $\mathrm{CO}(7-6)$ (purple), and [C I] (yellow) transitions $(\mathrm{CO}(4-3)$ is not available for this source). The solid red line indicates the best-fit Gaussian model. The black tick shows the expected line position based on the optical redshift $z_{\text {spec,opt }}$. The full compilation of SEDs and spectra from which we extracted reliable line measurements (Sect. 3.2.2) is available at http://doi .org/10.5281/zenodo. 3967380.

computed over progressively larger frequency windows centered on each channel. The line flux is then the weighted average flux density within the frequency interval maximizing the $\mathrm{S} / \mathrm{N}$, times the velocity range covered by the channels within the window, and minus the continuum emission. To estimate the latter, we considered a $\mathrm{S} / \mathrm{N}$-weighted average of line-free channels over the full spectral width $(\sim 7.5 \mathrm{GHz})$, assuming an intrinsic powerlaw shape $\left(\propto v^{3.7}\right)$. The redshift is determined from the weighted mean of the frequencies covered by the candidate line.

To confirm the line emission and avoid noise artifacts, we ran extensive simulations and computed the probability for each candidate line to be spurious $\left(P_{\text {line }}\right)$ following the approach in Jin et al. (2019). The calculation provides the chance that a candidate line with a known $\mathrm{S} / \mathrm{N}$, frequency, and velocity width appears in the spectrum owing to noise fluctuations, taking into account the full velocity range covered by the observations, the frequency sampling, and assuming a fixed range of acceptable line FWHM. As a further check, two members of our team visually inspected all the available spectra independently.

Once the redshift was determined, we finally remeasured the flux of each line over a fixed velocity width, normally set by $\mathrm{CO}(5-4)$ because of its brightness and the widespread availability, being the primary target of our survey. We rounded the velocity width to the closest integer number of channels allowed by the frequency resolution of each spectrum. The adoption of identical apertures for the spectral extractions and constant velocity ranges for the measurements allowed us to derive consistent line ratios and depict meaningful CO SLEDs. We also note that the redshift and the velocity width of every transition are fully consistent with the ones of $\mathrm{CO}(5-4)$, when they were left free to vary.

For sources without a significant flux detection, we estimated an upper limit around the expected line position. Whenever an alternative ALMA line measurement was available, we adopted $z_{\text {spec,submm }}$ and the known velocity width to set a $3 \sigma$ limit of $I_{\text {line }}>3 \times \mathrm{rms}_{\mathrm{ch}} \sqrt{\mathrm{d} v \Delta v}$, where $\mathrm{rms}_{\mathrm{ch}}$ is the average noise per channel over the line velocity width $\Delta v$, and $\mathrm{d} v$ is the velocity bin size. Such limits are reliable and useful, given the exact knowledge of the redshift from the submillimeter. When only a $z_{\text {spec,opt }}$ was available, we scanned the frequency around the expected location of the line, but eventually measured only upper limits adopting an arbitrary $\Delta v=400 \mathrm{~km} \mathrm{~s}^{-1}$ to be consistent with V18 and V20. Finally, we checked our blind flux measurements against a Gaussian parameterization of the line emission, resulting in a $\sim 10 \%$ flux difference due to the different velocity widths, and fully consistent redshift estimates, similarly to previous works (Coogan et al. 2018, V18). We accounted for this factor by increasing the line fluxes from the spectral scanning by $10 \%$. We note that a blind scanning is less prone to spurious detections and it provides more reliable estimates, whenever previous detailed knowledge of a source is absent. Therefore, we adopted the fluxes estimated by scanning the spectra as our final measurements.

As an example, we show the spectra of a source with multiple line detections in Fig. 2, along with its IR SED. The whole compilation of spectra from which we extracted reliable measurements is available online ${ }^{2}$.

\subsubsection{Quality flags and detection rate}

We finally classified the spectra and the $z_{\text {spec,submm }}$ estimate for each line by visual inspection and comparison with the optical and near-IR determinations.

- Flag $_{\text {line }}=1:$ Secure line measurement due to low probability of being a false positive $\left(P_{\text {spurious }}<0.01\right)$ and/or presence of alternative lines confirming the $z_{\text {spec,submm }}$, consistently with the optical or near-IR determination $z_{\text {spec,opt }}$.

- Flag $_{\text {line }}=0.5$ : Secure upper limit on the line flux, given the presence of alternative submillimeter lines confirming $z_{\text {spec,submm }}$.

- Flag line $=0$ : Upper limit on the line flux, assuming a velocity width of $\Delta v=400 \mathrm{~km} \mathrm{~s}^{-1}$ centered at the expected frequency based on a high quality $z_{\text {spec,opt }}$.

- Flag line $_{1}=-1$ : Undetected line and uncertain upper limit due to a poor quality $z_{\text {spec,opt }}$.

- Flag line $=-99:$ Line not observed (no data).

We consider "reliable" the flux measurements or upper limits for lines with Flag line $\geq 0.5$, and "uncertain" when Flag line $\leq 0$.

2 http://doi.org/10.5281/zenodo. 3967380 


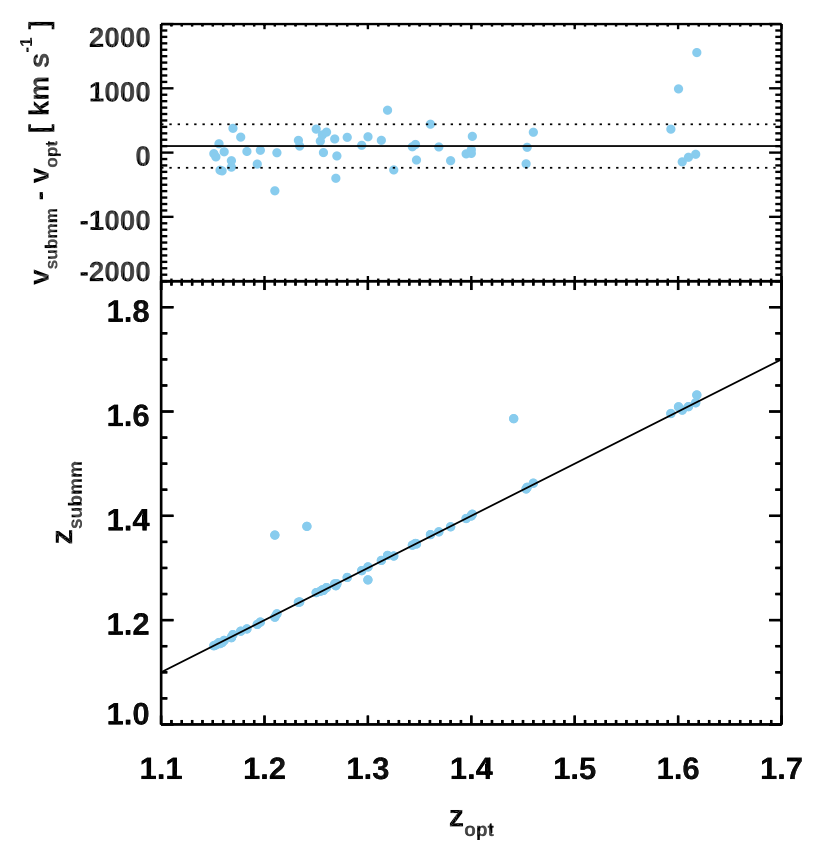

Fig. 3. Spectroscopic redshift offset between submillimeter and optical or near-IR observations. Bottom: the blue filled circles indicate the new $z_{\text {spec,submm }}$ determinations for trustable emission lines detected by ALMA against the optical or near-IR $z_{\text {spec,opt }}$ from the COSMOS master compilation (Salvato et al., in prep.). The solid black line marks the locus of $z_{\text {spec,submm }}=z_{\text {spec,opt }}$. Top: $v_{\text {submm }}-v_{\text {opt }}$ difference as a function of $z_{\text {spec,opt }}$, excluding three catastrophic strong outliers and an ascertained misidentification. The solid and dotted lines mark the mean and the $1 \sigma$ dispersion of the sample.

Adopting this scheme, we recovered 56/123 ( 46\%) sources with reliable $\mathrm{CO}(5-4)$ flux estimates for the primary sample, and 41/75 ( $55 \%)$ for $\mathrm{CO}(2-1)$. As foreseeable for targeted observations, we achieved higher detection rates for $\mathrm{CO}(7-6)$ and $[\mathrm{CI}]\left({ }^{3} P_{2}-{ }^{3} P_{1}\right)(13 / 15$ detections, $\sim 87 \%)$. Considering only sources with previous knowledge of $z_{\text {spec,submm }}$ rather than $z_{\text {spec,opt }}$, the detection rate jumps to $100 \%$.

In Appendix B we revisit a posteriori the selection of the targets for the $\mathrm{CO}(2-1)$ follow-up. This allows us to identify the factors setting the detection rate: the quality of $z_{\text {spec,opt }}$, lower $L_{\mathrm{IR}}$ than initially estimated, and intrinsically faint lines in bright objects, in order of importance. Here we remark that the imposition of minimum $I_{21}$ and $I_{54}$ flux thresholds formally biases their ratio. To account for this selection effect, when deriving average CO SLEDs later on, we will limit the calculation to objects that would have entered the sample of potential $\mathrm{CO}(2-1)$ targets according to the revised IR modeling.

\subsubsection{Spectroscopic redshift offset: submillimeter vs optical and near-infrared}

In Fig. 3 we show the comparison between the spectroscopic redshifts for the reliable ALMA sources and their original $z_{\text {spec,opt }}$ estimate from the master compilation of the COSMOS field (Salvato et al., in prep.). Excluding four sources with ascertained significant deviations $\left(z_{\text {spec,submm }}-z_{\text {spec,opt }}>0.05\right)$, the ALMA and the optical or near-IR redshift estimates are in good agreement with mean and median $\left(z_{\text {spec,submm }}-z_{\text {spec,opt }}\right)<0.001$ and a normalized median absolute deviation (Hoaglin et al. $1983)$ of $\left|z_{\text {spec,submm }}-z_{\text {spec,opt }}\right| /\left(1+z_{\text {spec,submm }}\right)=0.001$. The redshift difference corresponds to a mean velocity offset of
$\langle\Delta v($ submm $-\mathrm{opt})\rangle=101 \pm 46 \mathrm{~km} \mathrm{~s}^{-1}$ with a dispersion of $338 \mathrm{~km} \mathrm{~s}^{-1}$, as measured over 54 objects. The outliers have either dubious quality or no flags on $z_{\text {spec,opt }}$.

\subsubsection{Serendipitous detections}

We serendipitously found multiple sources in the dust continuum emission maps of 12 primary targets. Four physical pairs are spectroscopically confirmed by ALMA (Puglisi et al. 2019), while the remaining 8/12 are detected in continuum emission only. Five out of 6 reliable systems in this pool are well separated and deblended in both the $K$-band and in the far-IR. We flagged the only other object possibly affected by blending (\#51599) and adopted the total stellar mass and $L_{\mathrm{IR}}$ as representative of the whole system (see Puglisi et al. 2019) for a possible deblending of this source). We did not include the confirmed deblended companions any further in the analysis, in order to preserve the original selection. However, adding the only object that a posteriori meets our initial criteria would not change the main conclusions of this work.

\subsection{Infrared SED modeling}

We remodeled the IR photometry of our sources from the superdeblended catalog of the COSMOS field (Jin et al. 2018) in order to derive key physical properties of the sample, notably the total IR luminosity $L_{\mathrm{IR}}$, the dust mass $M_{\mathrm{dust}}$, and the mean intensity of the radiation field $\langle U\rangle$. Jin et al. (2018) chose radio and UltraVISTA $K_{s}$ priors to deblend the highly confused far-IR and submillimeter images, while performing active removal of nonrelevant priors via SED fitting with redshift information and Monte Carlo simulations on real maps, which reduces the degeneracies and results in well-behaved flux density uncertainties (Liu et al. 2018). The catalog includes emission recorded by Spitzer/MIPS at $24 \mu \mathrm{m}$ (Sanders et al. 2007), Herschel/PACS (Lutz et al. 2011) and SPIRE (Oliver et al. 2012) at 100-500 $\mu \mathrm{m}$, JCMT/SCUBA2 at $850 \mu \mathrm{m}$ (Geach et al. 2017), ASTE/AzTEC at $1.1 \mathrm{~mm}$ (Aretxaga et al. 2011), and IRAM/MAMBO at $1.2 \mathrm{~mm}$ (Bertoldi et al. 2007), plus complementary information at VLA/10 cm (3 GHz, Smolčić et al. 2017) and $21 \mathrm{~cm}(1.4 \mathrm{GHz}$, Schinnerer et al. 2010). Furthermore, we added to this list the information on the dust continuum emission that we measured with ALMA in the observed $0.8-3.2 \mathrm{~mm}$ range.

Our modeling follows the approach of Magdis et al. (2012) and V20. We used an expanded library of Draine \& Li (2007) models and the AGN templates from Mullaney et al. (2011) to estimate the total IR luminosity $L_{\mathrm{IR}}(8-1000 \mu \mathrm{m})-$ splitting the contribution from star-formation and dusty tori -, the dust mass $M_{\text {dust }}$, and the intensity of the radiation field $\langle U\rangle$. The latter is a dimensionless quantity that can be written as $\langle U\rangle=1 / 125 \times$ $L_{\mathrm{IR}} / M_{\text {dust }}$, the constant expressing the power absorbed per unit dust mass in a radiation field where $\langle U\rangle=1$ (Draine \& Li 2007; Magdis et al. 2012). Moreover, $\langle U\rangle$ can be directly related to a mass-weighted dust temperature $\left(\langle U\rangle=\left(T_{\text {dust,mass }} / 18.9 \mathrm{~K}\right)^{6.04}\right.$, Magdis et al. 2017). The mass-weighted $T_{\text {dust,mass }}$ is $\sim 10 \%$ lower than the light-weighted estimate (Schreiber et al. 2018).

\subsection{AGN contamination}

Each best-fit IR SED model bears a fraction of the total luminosity due to dusty tori surrounding central AGN: $f_{\mathrm{AGN}}=$ $L_{\mathrm{IR}, \mathrm{AGN}} / L_{\mathrm{IR}}$, with $L_{\mathrm{IR}}=L_{\mathrm{IR}, \mathrm{AGN}}+L_{\mathrm{IR}, \mathrm{SFR}}$. Clearly, the ability to detect the AGN emission critically depends on the coverage of the mid-IR wavelengths and the intrinsic brightness of the dust 
F. Valentino et al.: $\mathrm{CO}$ emission in distant main-sequence galaxies

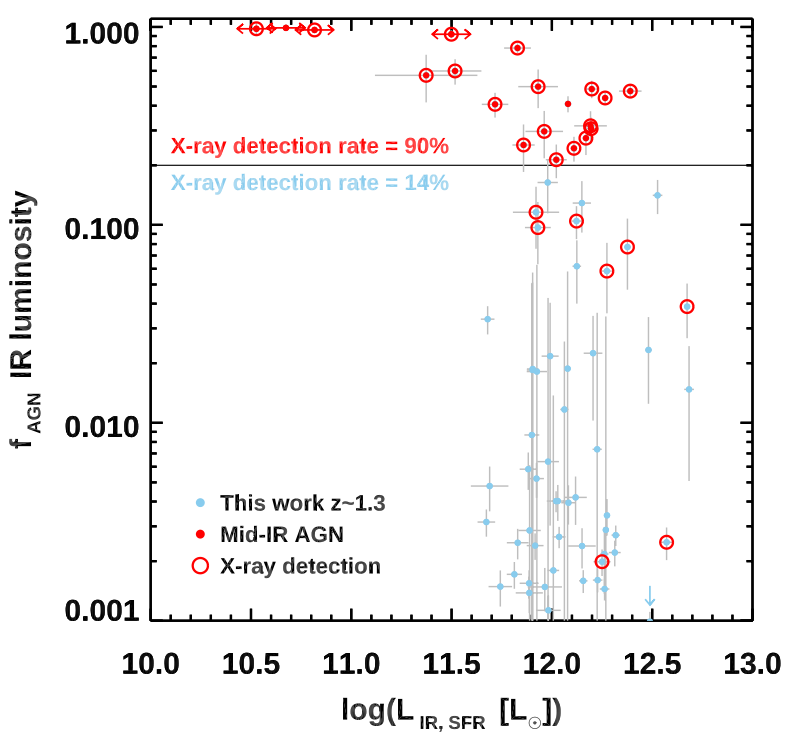

Fig. 4. AGN contamination. Fraction of the AGN contribution to the total IR luminosity $\left(f_{\mathrm{AGN}}=L_{\mathrm{IR}, \mathrm{AGN}} / L_{\mathrm{IR}}\right)$ as a function of the IR luminosity from the star-forming component of the SED fitting $\left(L_{\mathrm{IR}, \mathrm{SFR}}\right)$. The blue points show the objects with a line emission or an upper limit (Flag $\geq 0$ ) and a good model of the SED. Red solid circles indicate mid-IR selected AGN. Red empty circles indicate Chandra $2-10 \mathrm{keV}$ detections with $L_{2-10 \mathrm{keV}} \geq 10^{42} \mathrm{erg} \mathrm{s}^{-1}$. Horizontal red arrows indicate sources with unreliable estimates of $L_{\mathrm{IR}, \mathrm{SFR}}$ due to the strong AGN contamination.

surrounding the nuclei. We, thus, consider reliably detected the contribution from an AGN when $f_{\mathrm{AGN}}+1 \sigma_{f_{\mathrm{AGN}}} \geq 20 \%$, while galaxies with $f_{\mathrm{AGN}} \geq 80 \%$ are flagged as AGN dominated. Estimates of $f_{\mathrm{AGN}} \lesssim 1 \%$ simply indicate the absence of strong AGN components and they should not be taken at face value. For the sake of completeness, in Fig. 4 we show their statistical uncertainty $\sigma_{f_{\mathrm{AGN}}}$ associated with the fitting procedure.

According to our scheme, we find AGN signatures in $~ 30 \%$ of the sample, as previously reported (Puglisi et al. 2019). A similar SED-based classification largely overlaps with Spitzer/IRAC color criteria widely used in the literature (Donley et al. 2012, V20). Figure 4 further shows how our AGN scheme overlaps with the detection rate of hard X-ray photons from Chandra (Marchesi et al. 2016; Civano et al. 2016). Ninety percent of sources with Flag $\geq 0$ and $f_{\mathrm{AGN}}+1 \sigma_{f_{\mathrm{AGN}}} \geq 20 \%$ also have $L_{2-10 \mathrm{keV}} \geq 10^{42} \mathrm{erg} \mathrm{s}^{-1}$. This fraction drops to $14 \%$ below the fixed $f_{\mathrm{AGN}}$ threshold, which might be considered as the rate of AGN contamination in our star-forming dominated sample. Here we limit the analysis to this classification and to the quantification of the AGN contribution to the total IR luminosity. We note that we exclude the AGN-dominated galaxies from the rest of the analysis. A specific study of the effects of the AGN on the gas excitation is postponed to the future.

\subsection{Ancillary data}

We took advantage of the rich ancillary data and past analysis available for the COSMOS field by compiling stellar masses (Muzzin et al. 2013; Laigle et al. 2016) and optical and nearIR spectroscopic redshifts (Salvato et al., in prep.). For sources with X-ray counterparts and a substantial AGN contamination, we refit the UV to near-IR photometry using the code $\mathrm{CIGALE}^{3}$ (Noll et al. 2009), self-consistently accounting for the presence

\footnotetext{
3 https://cigale.lam.fr/
}

Table 2. CO emission from literature data.

\begin{tabular}{lcc}
\hline \hline Sample & Transition & References \\
\hline Herschel-FTS archive & $J=1,2$ & K16 \\
& $J=5,7$ & L15a \\
HerCULES & $J=5$ & R15, L15a \\
HERACLES & $J=2,5$ & L08; L+in prep. \\
PHIBSS-2 & $J=2$ & F19 \\
BzK $z \sim 1.5$ & $J=1$ & A10; A14 \\
& $J=2$ & D10a \\
Starburst $z \sim 1.5$ & $J=3,5$ & Da09, D15 \\
& $J=2,3$ & S15, S18b; \\
SMGs & $J=5$ & S18a \\
\hline
\end{tabular}

Notes. ${ }^{(a)}$ We refer the reader to Sect. 3.6 of this work, V18, and V20 for detailed references for the SMG population.

References. K16: Kamenetzky et al. (2016); L15a: Liu et al. (2015a); R15: Rosenberg et al. (2015); L08: Leroy et al. (2008); L+ in prep.: Liu et al. (in prep.); F19: Freundlich et al. (2019); A10: Aravena et al. (2010); A14: Aravena et al. (2014); D10: Daddi et al. (2010a); Da09: Dannerbauer et al. (2009); D15: Daddi et al. (2015); S15: Silverman et al. (2015); S18a,b: Silverman et al. (2018a,b); V18: Valentino et al. (2018); V20: Valentino et al. (2020).

of emission from the active nuclei across the various bands as detailed in Circosta et al. (2018). While this provided us with a more robust estimate of the stellar masses in presence of strong AGN, we did not find any significant offset between $M_{\star}$ from CIGALE and the COSMOS catalogs for the rest of the sample.

\subsection{Literature data}

To put in context the new ALMA data, we compiled samples from the literature (Table 2). For what concerns objects in the local Universe, we included the local IR-bright galaxies from the full Herschel-FTS archive and their ancillary observations (Liu et al. 2015a; Kamenetzky et al. 2016; Lu et al. 2017, see also V20), covering both low- and high- $J$ CO transitions. We further added the (U)LIRGs from the HerCULES survey (Rosenberg et al. 2015), and the local spirals from the HERACLES (Bigiel et al. 2008; Leroy et al. 2008, 2009) and KINGFISH surveys (Kennicutt et al. 2011; Dale et al. 2012, 2017). We note that other collections of nearby objects with coverage of low- $J$ CO emission are available (e.g., Cicone et al. 2017; Saintonge et al. 2017; Pan et al. 2018; Gao et al. 2019), but we privileged galaxies with observables and properties more directly comparable with our ALMA sample.

At higher redshifts, we incorporated the MS and SB observations from the PHIBSS-2 survey at $z=0.5-0.8$ (Freundlich et al. 2019), the four $B z K$ galaxies in D15 (Dannerbauer et al. 2009; Daddi et al. 2010a; Aravena et al. 2010, 2014), and a pool of starbursts at $z=1.5$ (Silverman et al. 2015, 2018a,b). Finally, we included samples of the high-redshift submillimeter galaxies and quasars (Walter et al. 2011; Alaghband-Zadeh et al. 2013; Aravena et al. 2016; Bothwell et al. 2017; Yang et al. 2017; Andreani et al. 2018; Cañameras et al. 2018; Liu et al. 2015a; Carilli \& Walter 2013, see V18 and V20).

For the whole compilation, we homogenized the measurements to our assumptions (stellar IMF, cosmology, far-IR modeling). Whenever publicly available, we refit the far-IR photometry adopting the same recipes as described in Sect. 3.3 to 
avoid systematics. This is the case for the subsample of the PHIBSS-2 survey in COSMOS and the compilation described in V18 and V20. We used the total $L_{\mathrm{IR}}$ reported in Whitaker et al. (2014) for the remaining fields covered by PHIBSS-2. Since a similar approach to ours has been used to model the SED of $B z K$ s and SBs at $z \sim 1.5$, we adopted the best-fit values reported in the original papers. As detailed in V20, for the local sample of IR-bright galaxies we converted the IRAS-based 40-400 $\mu \mathrm{m}$ farIR luminosities (Sanders et al. 2003) to total estimates integrated

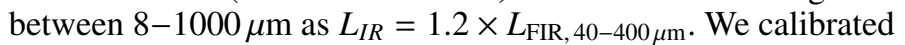
this factor on a subsample of galaxies from the Great Observatories All-Sky LIRGs Survey (GOALS, Armus et al. 2009). Finally, whenever necessary, we increased by a factor of $1.5 \times$ the total $L_{\mathrm{IR}}$ from the modified black body modeling to match the values from Draine \& Li (2007) templates (Magdis et al. 2012).

\section{Results}

\subsection{How the CO emission correlates with the infrared luminosity}

In Fig. 5 we show how low-, mid-, and high- $J$ CO $L^{\prime}$ luminosities compare with the infrared luminosities $L_{\mathrm{IR}}$, including both our new ALMA data on distant main-sequence and starburst galaxies and the literature sample. Note that we inverted the axes with respect to the canonical representation of the star formation laws in order to facilitate the comparison between the various tracers and across different figures. Here we adopted the $L_{\mathrm{IR}}$ corrected for the contribution of the dusty tori surrounding the AGN, excluding those sources dominated by such contribution $\left(f_{\mathrm{AGN}} \geq 0.8\right.$, Sect. 3.4) or well-known QSOs at high redshift. These objects tend to increase the scatter of the relation, being overluminous in the mid-IR portion of their SED.

The $L^{\prime}$ luminosity of each CO transition strongly correlates with $L_{\mathrm{IR}}$. The upper left panel of Fig. 5 shows that the $\mathrm{CO}(2-1)$ emission in our main-sequence and starburst galaxies is consistent with the two-mode star formation model described in Sargent et al. (2014), where both samples follow a sublinear relation with different normalizations $\left(\log \left(L_{\mathrm{CO}(1-0)}^{\prime}\right)=0.81 \times\right.$ $\log \left(L_{\mathrm{IR}}\right)$, corresponding to a superlinear slope of 1.23 in the canonical SK plane). Here we applied to the model tracks a $L_{\mathrm{CO}(1-0)}^{\prime} / L_{\mathrm{CO}(2-1)}^{\prime}=1.2$ excitation correction for $B z K$-selected galaxies (Daddi et al. 2010a, D15), but similar considerations hold for the populations of starbursting objects and SMGs.

On the other hand, applying a Bayesian regression analysis (LINMIX _ERR.PRO, Kelly 2007):

$\log \left(\frac{L_{\mathrm{CO}, J}^{\prime}}{\mathrm{Kkm} \mathrm{s}^{-1} \mathrm{pc}^{2}}\right)=\alpha+\beta \times \log \left(\frac{L_{\mathrm{IR}}}{L_{\odot}}\right)$

returns $\beta \sim 1$ for both $J=5,7$ (Table 3). Similarly to what is known for local IR-bright and high-redshift SMGs (Greve et al. 2014; Liu et al. 2015a; Lu et al. 2015; Kamenetzky et al. 2016, $\mathrm{D} 15)$, this proves that mid- and high- $J$ CO luminosities of distant main-sequence and starburst galaxies follow the same tight linear correlation with the total $L_{\mathrm{IR}}$, suggesting that these transitions might be used as SFR - rather than molecular $M_{\text {gas }}$ - tracers. This also suggests caution when deriving the total molecular $M_{\text {gas }}$ from high- $J$ CO transitions without prior knowledge of the excitation conditions. The modeling includes our and literature sources with $L_{\mathrm{IR}} \geq 10^{8.5} L_{\odot}$, reliable upper limits on the line luminosities (Flag $=0.5)$, uncertainties on both variables, and it excludes AGN-dominated galaxies or QSOs. However, given the large dynamical range spanned by the observations and the small sample of bright QSOs and upper limits, the best-fit models are largely unaffected by their inclusion. The observed points are similarly scattered around the best fit relations for $\mathrm{CO}(5-4)$ and $\mathrm{CO}(7-6)$, with an intrinsic scatter of 0.16 dex. We note that excluding the SMGs from the fitting reduces the scatter of the $L_{\mathrm{IR}}-L_{\mathrm{CO}(7-6)}^{\prime}$ relation to $0.14 \mathrm{dex}$, but not of the $L_{\mathrm{IR}}-L_{\mathrm{CO}(5-4)}^{\prime}$ correlation, leaving unaltered their slopes. This is consistent with previous determinations of the scatter of the $L_{\mathrm{CO}, J}^{\prime}-L_{\mathrm{IR}}$ relations based on local IR-bright objects only, which found the $J=7$ luminosity to form the tightest relation with $L_{\mathrm{IR}}$ (e.g., Liu et al. 2015a). On the other hand, fitting only the high-redshift samples changes the slopes of the two relations by $<15 \%$ at $<3 \sigma$ significance. We note that the inclusion of the low-redshift samples drives the difference between our regression analysis of $L_{\mathrm{IR}}-L_{\mathrm{CO}(5-4)}^{\prime}$ and that of D15.

\subsection{CO emission and excitation on and above the main sequence}

The homogeneous IR-selection of galaxies presented above allows us to explore the $\mathrm{CO}$ emission and the excitation properties over a wide range of distances from the main sequence $\left(\triangle \mathrm{MS}=\mathrm{SFR} / \mathrm{SFR}_{\mathrm{MS}}\right)$. This is what is shown in Fig. 6 , where we report the trend of $L^{\prime} / L_{\mathrm{IR}}$ ratios and a proxy for the $\mathrm{CO}$ excitation $\left(R_{52}=L_{\mathrm{CO}(5-4)}^{\prime} / L_{\mathrm{CO}(2-1)}^{\prime}\right)$ as a function of the position with respect to the main sequence, parameterized as in Sargent et al. (2014). Here we consider only the dust-obscured SFR traced by $L_{\mathrm{IR}}$, without including the contribution from UV emission. The latter becomes of lesser importance in massive SFGs and at increasing redshifts, as for the samples explored here, but this simplification does not apply to local and low $M_{\star}$ objects. We, thus, use galaxy samples with well determined $L_{\mathrm{IR}}$ for our comparison.

\subsubsection{The low-J transition}

The $L_{\mathrm{CO}(2-1)}^{\prime} / L_{\mathrm{IR}}$ ratio constantly declines for increasing $\Delta \mathrm{MS}$, a well-known tendency generally ascribable to the shorter depletion timescales and higher SFEs of starburst galaxies than main-sequence objects (e.g., Daddi et al. 2010b; Tacconi et al. 2010, 2018, 2020; Genzel et al. 2015; Saintonge et al. 2017; Freundlich et al. 2019; Liu et al. 2019a). A linear regression analysis confirms the existence of a meaningful anticorrelation between $\Delta \mathrm{MS}$ and $L_{\mathrm{CO}(2-1)}^{\prime} / L_{\mathrm{IR}}$ (Fig. 6). However, we note that the sublinear $L_{\mathrm{IR}}-L_{\mathrm{CO}(2-1)}^{\prime}$ relation (Fig. 5), coupled with the higher $L_{\mathrm{IR}}$ at fixed $M_{\star}$ for distant main-sequence galaxies, introduces a redshift dependence in the $\Delta \mathrm{MS}-L_{\mathrm{CO}(2-1)}^{\prime} / L_{\mathrm{IR}}$ relation, which reflects the increasing SFE with redshift (Magdis et al. 2012). The magnitude of this effect in the range $z=0.5-1.5 \mathrm{cov}-$ ered by the PHIBSS-2 and our sample can be gauged by the shift of the tracks from the two-mode star formation model by Sargent et al. (2014), based on the relations shown in Fig. 5. The tracks are calculated for $M_{\star}=10^{10.7} M_{\odot}$, the median stellar mass of both samples, and were calibrated against the data available at that time, which did not include a significant population of starbursts. When fitting separately our $z \sim 1.3$ and the PHIBSS-2 samples at $z=0.5-0.8$, we retrieve a similar displacement. The slopes are similar and consistent with the shallow increase of the SFE along the main sequence reported by Sargent et al. (2014), but we do not detect any abrupt change when entering the starburst regime. 

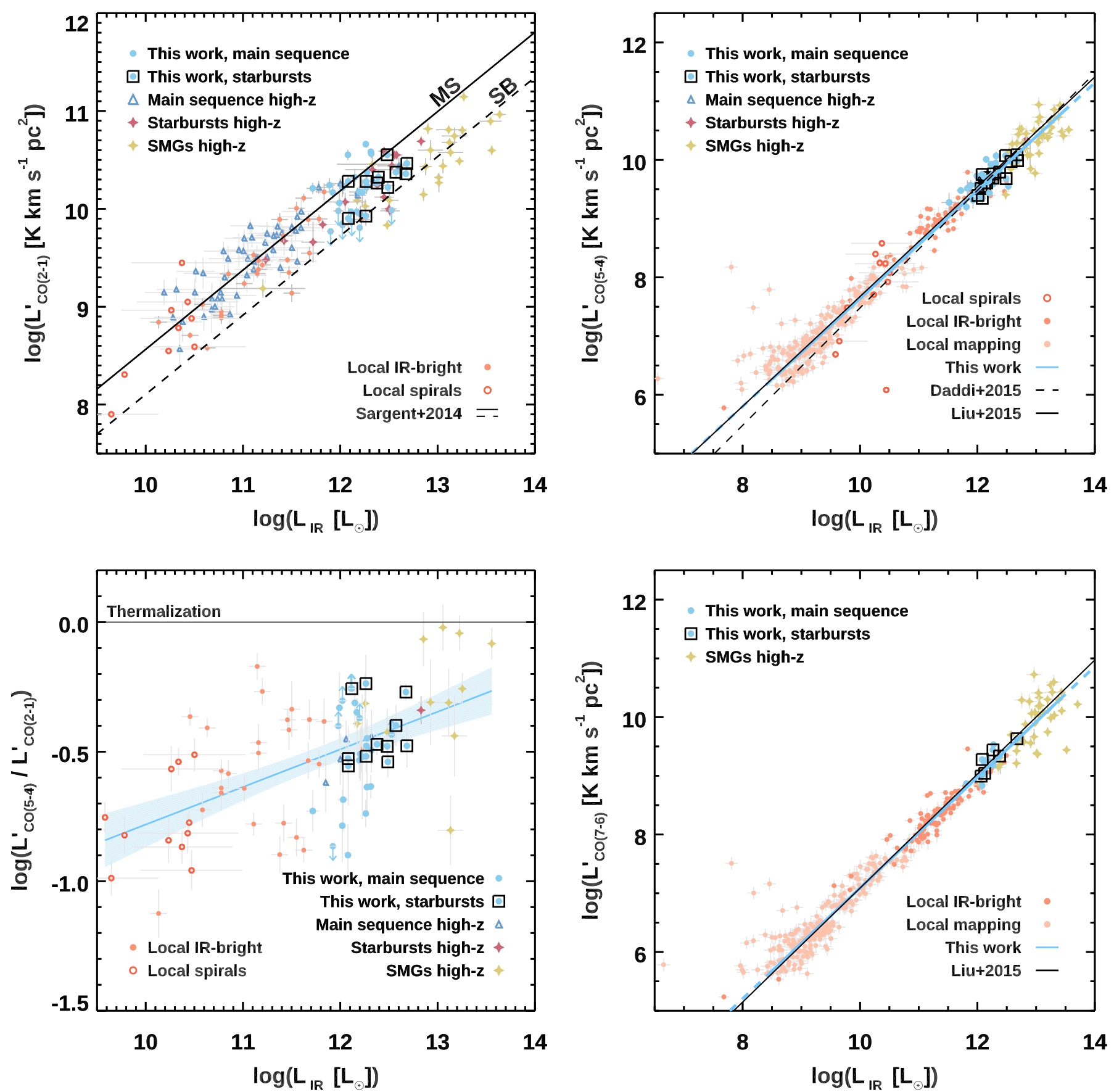

Fig. 5. $\mathrm{CO}$ - IR luminosities relations. From the top left, clockwise: $L^{\prime}\left[\mathrm{K} \mathrm{km} \mathrm{s}^{-1} \mathrm{pc}^{2}\right]$ luminosities of $\mathrm{CO}(2-1), \mathrm{CO}(5-4), \mathrm{CO}(7-6)$, and the $\mathrm{CO}(5-4) / \mathrm{CO}(2-1)$ ratio as a function of the IR luminosity $L_{\mathrm{IR}}\left[L_{\odot}\right]$. Symbols are color-coded as labeled (see Sect. 3.6 for references), where our samples of main-sequence galaxies and starbursts $(\Delta \mathrm{MS} \geq 3.5)$ at $z \sim 1.3$ are marked by blue filled circles and open black squares, respectively. The solid and dashed black lines show the best-fit linear models from previous works (Sargent et al. 2014; Liu et al. 2015a, D15). The blue lines mark our best model, where the dashed segments indicate its extrapolation. The blue shaded area in the bottom left figure shows the 95\% confidence interval, omitted in the remaining panels for clarity. The best-fit parameters and the scatter are reported in Table 3 . We adopted an extended $L_{\mathrm{IR}}$ range in the right panels to show the $L_{\mathrm{CO}(5-4)}^{\prime}$ and $L_{\mathrm{CO}(7-6)}^{\prime}$ luminosities obtained from mapping nearby objects (Liu et al. 2015a), for which $\mathrm{CO}(2-1)$ observations on the same scales are not available. Note that the axes are inverted with respect to the canonical representation of star formation laws.

\subsubsection{The mid- and high-J $\mathrm{CO}$ transitions}

On the other hand, both the $L_{\mathrm{CO}(5-4)}^{\prime} / L_{\mathrm{IR}}$ and $L_{\mathrm{CO}(7-6)}^{\prime} / L_{\mathrm{IR}}$ ratios are constant as a function of $\Delta \mathrm{MS}$ (Table 3 ), following the linear $L_{\mathrm{IR}}-L_{J=57}^{\prime}$ correlation shown in Fig. 5 . This strengthens the idea that mid- and high- $J$ transitions do trace the SFR, rather than the total molecular $M_{\text {gas }}$ in galaxies, and they do so independently of their stellar mass and redshift, within the parameter space of massive and metal-rich objects spanned by the observations presented here. Then, the $R_{52}$ ratio naturally rises as the distance from the main sequence increases: the $\mathrm{CO}$ emission in starburst galaxies appears more excited than in main-sequence 
Table 3. Scaling relations involving the $\mathrm{CO}$ emission lines.

\begin{tabular}{lcccc}
\hline \hline Relation $\left.{ }^{\dagger}\right)$ & Slope & Intercept & Intrinsic scatter & Correlation \\
$x, y$ & $\beta$ & $\alpha$ & $\sigma_{\text {int }}$ & $\rho$ \\
\hline \multicolumn{4}{c}{ Luminosities } \\
\hline$L_{\mathrm{IR}}, L_{\mathrm{CO}(5-4)}^{\prime}$ & $0.92 \pm 0.01$ & $-1.55 \pm 0.08$ & $0.17 \pm 0.01$ & 0.99 \\
$L_{\mathrm{IR}}, L_{\mathrm{CO}(7-6)}^{\prime}$ & $0.94 \pm 0.01$ & $-2.34 \pm 0.09$ & $0.16 \pm 0.01$ & 0.99 \\
$L_{\mathrm{IR}}, R_{52}$ & $0.15 \pm 0.02$ & $-2.23 \pm 0.28$ & $0.17 \pm 0.02$ & 0.62 \\
\hline \multicolumn{5}{c}{ Distance from the main sequence } \\
\hline $\mathrm{MS}, L_{\mathrm{CO}(2-1)}^{\prime} / L_{\mathrm{IR}}{ }^{(\ddagger)}$ & $-0.34 \pm 0.13$ & $-1.84 \pm 0.08$ & $0.24 \pm 0.04$ & -0.50 \\
$\Delta \mathrm{MS}, L_{\mathrm{CO}(5-4)}^{\prime} / L_{\mathrm{IR}}$ & $-0.01 \pm 0.11$ & $-2.51 \pm 0.07$ & $0.33 \pm 0.03$ & -0.01 \\
$\Delta \mathrm{MS}, L_{\mathrm{CO}(7-6)}^{\prime} / L_{\mathrm{IR}}{ }^{(\ddagger)}$ & $0.08 \pm 0.18$ & $-3.02 \pm 0.11$ & $0.18 \pm 0.06$ & 0.18 \\
$\Delta \mathrm{MS}, R_{52}^{(\ddagger)}$ & $0.23 \pm 0.10$ & $-0.64 \pm 0.06$ & $0.13 \pm 0.03$ & 0.55 \\
\hline \multicolumn{3}{c}{ Drivers of the CO excitation } & \\
\hline$\langle U\rangle, R_{52}$ & $0.36 \pm 0.05$ & $-0.95 \pm 0.07$ & $0.15 \pm 0.02$ & 0.75 \\
$\mathrm{SFE}\left(Z=Z_{\mathrm{FMR}}\right), R_{52}$ & $0.37 \pm 0.05$ & $-0.66 \pm 0.03$ & $0.11 \pm 0.03$ & 0.88 \\
$\mathrm{SFE}\left(Z=Z_{\odot}\right), R_{52}$ & $0.31 \pm 0.04$ & $-0.68 \pm 0.03$ & $0.11 \pm 0.02$ & 0.89 \\
$\mathrm{SFE}\left(Z=Z_{\mathrm{FMR}} \vee Z_{\text {super }}\right), R_{52}$ & $0.32 \pm 0.04$ & $-0.69 \pm 0.03$ & $0.12 \pm 0.02$ & 0.86 \\
$\Sigma_{\mathrm{SFR}}, R_{52}$ & $0.16 \pm 0.02$ & $-0.60 \pm 0.03$ & $0.14 \pm 0.02$ & 0.82 \\
\hline
\end{tabular}

Notes. ${ }^{(\dagger)}$ The linear regression is applied to $\log$-quantities: $\log (y)=\alpha+\beta \times \log (x)$. The model accounts for errors on both variables and censored

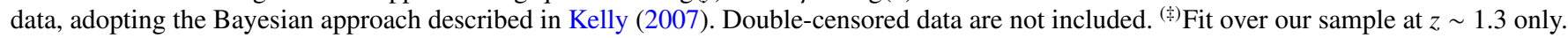

objects at similar stellar masses and redshifts (Table 3 ). As for $L_{\mathrm{CO}(2-1)}^{\prime} / L_{\mathrm{IR}}$, this relation is expected to evolve with redshift, mimicking the decrease of SFE over cosmic time. A separate fit for the local and the $z \sim 1.3$ galaxies seems to suggest this evolution, even if the small statistics of objects with both $\mathrm{CO}(2-1)$ and $\mathrm{CO}(5-4)$ lines available, especially on the lower main sequence, makes the $\Delta \mathrm{MS}-R_{52}$ trend more uncertain. The correlations are robust against the exclusion of the strongest outliers (Fig. 6). We note that the presence of sources on the MS with large ratios blurs the difference with SBs (see also Puglisi et al. 2019). A diversity of gas excitations conditions even among MS galaxies is evident.

\subsection{Main physical drivers of the CO excitation}

We now explore the relation between a proxy of the CO excitation conditions - the $R_{52}$ ratio - and a few physical quantities potentially steering the molecule's excitation: the star formation efficiency $\left(\mathrm{SFE}=\mathrm{SFR} / M_{\text {gas }}\right)$, its combination with the metallicity as probed by the mean interstellar radiation field intensity heating the dust $(\langle U\rangle \propto \mathrm{SFE} / Z)$, and the star formation surface density $\left(\Sigma_{\mathrm{SFR}}\right.$, Fig. 7$)$. Since we cannot spatially resolve the $\mathrm{CO}$ emission in our targets over many beams, this comparison applies to global galaxy scales. By complementing our measurements with the existing literature, we can span a wide interval of redshifts, masses, SFRs, and ISM conditions. The addition of a few tens of main-sequence and starburst galaxies further allows us to derive the average trends among different quantities and to explore their scatter.

\subsubsection{The star formation efficiency and the mean interstellar radiation field intensity}

By homogeneously modeling the far-IR SEDs of our sample and objects from the literature (Sect. 3.3), we retrieve sublinear correlations between $\langle U\rangle$ or $\mathrm{SFE}$ and $L_{\mathrm{CO}(5-4)}^{\prime} / L_{\mathrm{CO}(2-1)}^{\prime}$, as previously reported by D15. For our own sample of ALMA detections and reliable upper limits, we calculated SFE by converting the $M_{\text {dust }}$ from the SED fitting with Draine \& Li (2007) models into $M_{\text {gas }}$ applying a metallicity dependent gas-todust ratio $\log \left(\delta_{\mathrm{GDR}}(Z)\right)=10.54-0.99 \times(12+\log (\mathrm{O} / \mathrm{H}))$ (Magdis et al. 2012), assuming that galaxies on the main sequence follow a fundamental mass-metallicity relation (FMR, Mannucci et al. 2010). To be consistent with our previous work, we then assumed that starburst galaxies have supersolar metallicities $\left(\delta_{\mathrm{GDR}} \sim 30\right.$, while for reference $\delta_{\mathrm{GDR}} \sim 85$ for $Z=Z_{\odot}$, Magdis et al. 2012, see also Puglisi et al. 2017). We factored the 0.2 dex dispersion of the assumed mass-metallicity relation into the uncertainty of SFE. As a consistency check, we also modeled the SFE assuming a $\delta_{\mathrm{GDR}}(Z)$ with $Z=Z_{\mathrm{FMR}}\left(M_{\star}, \mathrm{SFR}\right)$ and $Z=Z_{\odot}$ for every galaxy in our sample, on and above the main sequence, retrieving consistent results within the uncertainties. We applied the same prescriptions to the literature data, considering SMGs as starbursting galaxies. This exacerbates the differences among observables (or, at least, parameters closer to measurements) when comparing starbursts and main-sequence galaxies. We warn the reader that these are well documented uncertainties on the use of dust as a molecular gas tracer (Magdis et al. 2012; Groves et al. 2015; Scoville et al. 2016), but similar considerations apply to $\mathrm{CO}$ and its $\alpha_{\mathrm{CO}}$ conversion factor (Bolatto et al. 2013). The choice of using dust instead of $\mathrm{CO}(2-1)$ to derive $M_{\text {gas }}$ was dictated by the attempt to reduce the correlation with the quantity under scrutiny, $R_{52}$.

The degeneracy on SFE driven by the $\delta_{\mathrm{GDR}}$ is partially alleviated when using $\langle U\rangle$ (Fig. 7). $\langle U\rangle$ carries similar information to SFE, mapped through an assumption on the metallicity $(\langle U\rangle \propto \mathrm{SFE} / Z)$. However, it does not imply an unknown conversion, since $\langle U\rangle \sim L_{\mathrm{IR}} / M_{\text {dust }}$, while still prone to assumptions as the optical depth of the dust emission (see Sect. 4.4.5). As clear from Fig. 7, starbursts and SMGs tend to display larger $\langle U\rangle$ and $\mathrm{CO}$ line ratios than main-sequence galaxies and local spirals, but the distinction in $\langle U\rangle$ is more blurred than in SFE. For reference, we also show the mean location of local spirals, ULIRGs, and $B z K$ s at $z \sim 1.5$ from D15. The linear regression analysis in the logarithmic space (Table 3 ) returns sublinear trends as in D15, but pointing toward a $1.7 \times$ smaller slope and with a larger intrinsic scatter (0.11-0.15 dex, Table 3). 

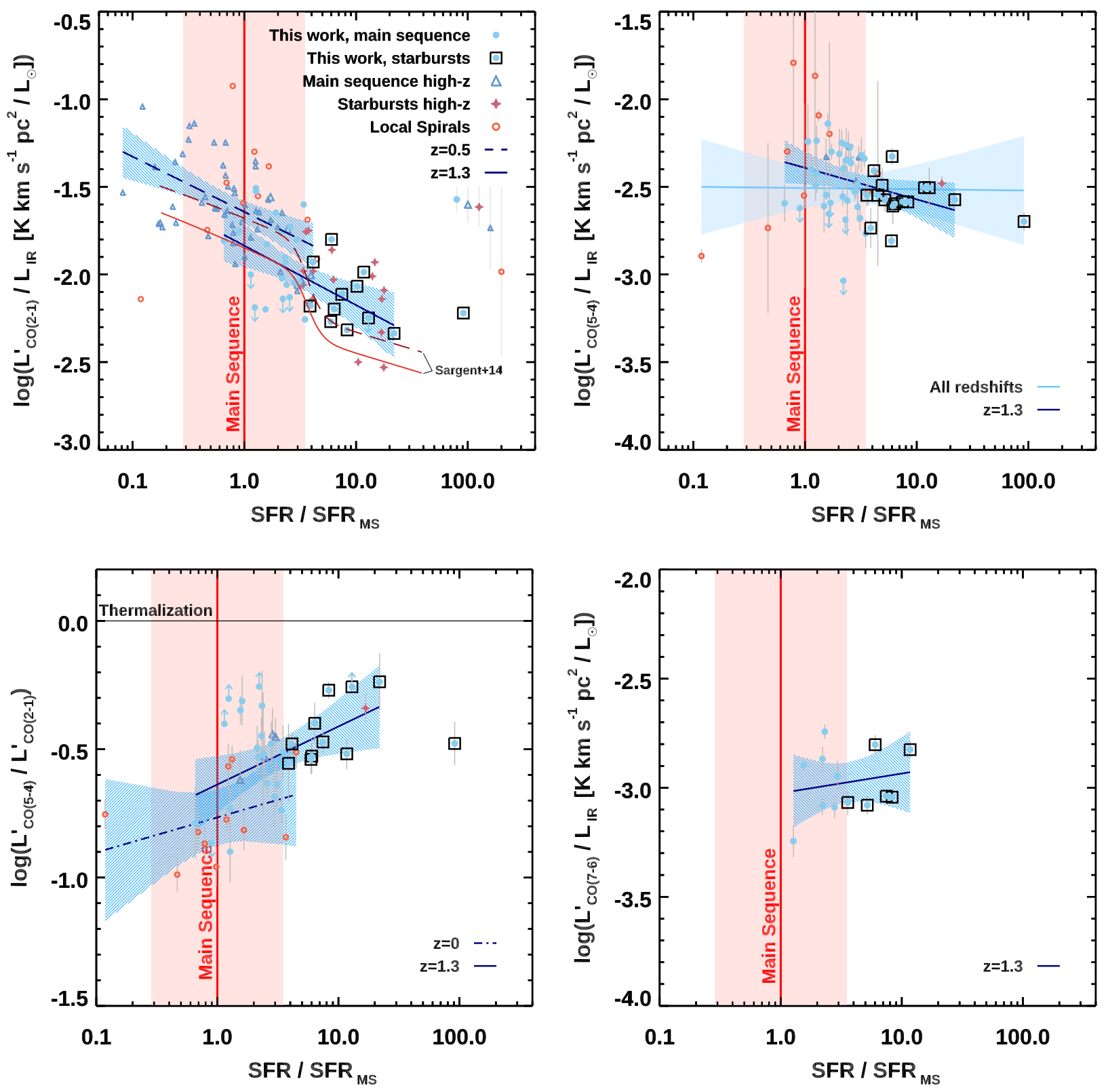

Fig. 6. $\mathrm{CO}$ emission on and above the main sequence. $L^{\prime}\left[\mathrm{K} \mathrm{km} \mathrm{s}^{-1} \mathrm{pc}^{2}\right] / L_{\mathrm{IR}}\left[L_{\odot}\right]$ for $\mathrm{CO}(2-1)$ (top left), $\mathrm{CO}(5-4)($ top right $)$, $\mathrm{CO}(7-6)$ (bottom right), and the $L_{\mathrm{CO}(5-4)}^{\prime} / L_{\mathrm{CO}(2-1)}^{\prime}$ ratio (bottom left) as a function of the distance from the main sequence. In every panel, the sample of main-sequence galaxies and starbursts at $z \sim 1.3$ presented here is marked by blue filled circles and open black squares. Open blue triangles and purple stars indicate other distant MS galaxies and SBs (at $z=0.5-0.8$ from the PHIBSS-2 survey of CO $(2-1)$, Freundlich et al. 2019; at $z \sim 1.5$ from D15, Silverman et al. 2018a,b). Open red circles signpost local spirals (Leroy et al. 2008; Kennicutt et al. 2011; Dale et al. 2012, 2017, Liu et al., in prep.). The red solid line and the shaded area indicate the main sequence within $\|\Delta \mathrm{MS}\|<3.5$ as parameterized by Sargent et al. (2014). The tracks and the shaded areas mark the best-fit models from the linear regression analysis and their $95 \%$ confidence interval when modeling the following samples: solid dark blue line: our galaxies at $z \sim 1.3$, excluding the strongest outlier above the main sequence; dashed dark blue line: PHIBSS-2 galaxies at $z=0.5-0.8$; dotted-dashed dark blue line: local spirals; solid light blue line: all samples available (see Table 3 for the parameters). The dashed and solid red lines in the top left panel show the tracks for the two-mode star formation model by Sargent et al. (2014) at $z=0.5$ and 1.5 and for $M_{\star}=10^{10.7} M_{\odot}$, the median mass of both the PHIBSS-2 and our sample. The median error bars for each sample are displayed in the panel.

\subsubsection{The SFR surface density}

The right panel of Fig. 7 shows the relation between $\Sigma_{\mathrm{SFR}}$ and $R_{52}$. For each object, we computed $\Sigma_{\mathrm{SFR}}=\mathrm{SFR} /\left(2 \pi R^{2}\right)$, where $R$ is a representative value of the galaxy radius. The latter is rather arbitrary and it depends on the chosen tracer, the depth, resolution, and wavelength of the observations. Here we adopted the ALMA sizes from circular Gaussian fitting for our sample, assuming $R=\mathrm{FWHM} / 2$. As mentioned in Sect. 3.2, this estimate combines all the available lines and continuum measurements, resulting in a size representative of the dust and gas content of each galaxy (Puglisi et al. 2019). We further recomputed the $\Sigma_{\mathrm{SFR}}$ for the $B z K$ galaxies in D15, using the Gaussian best-fit results of the rest-frame UV observations to be consistent with our estimates. For the SPT-SMGs, we used the sizes of Spilker et al. (2016), while we employed the $1.4 \mathrm{GHz}$ radio 


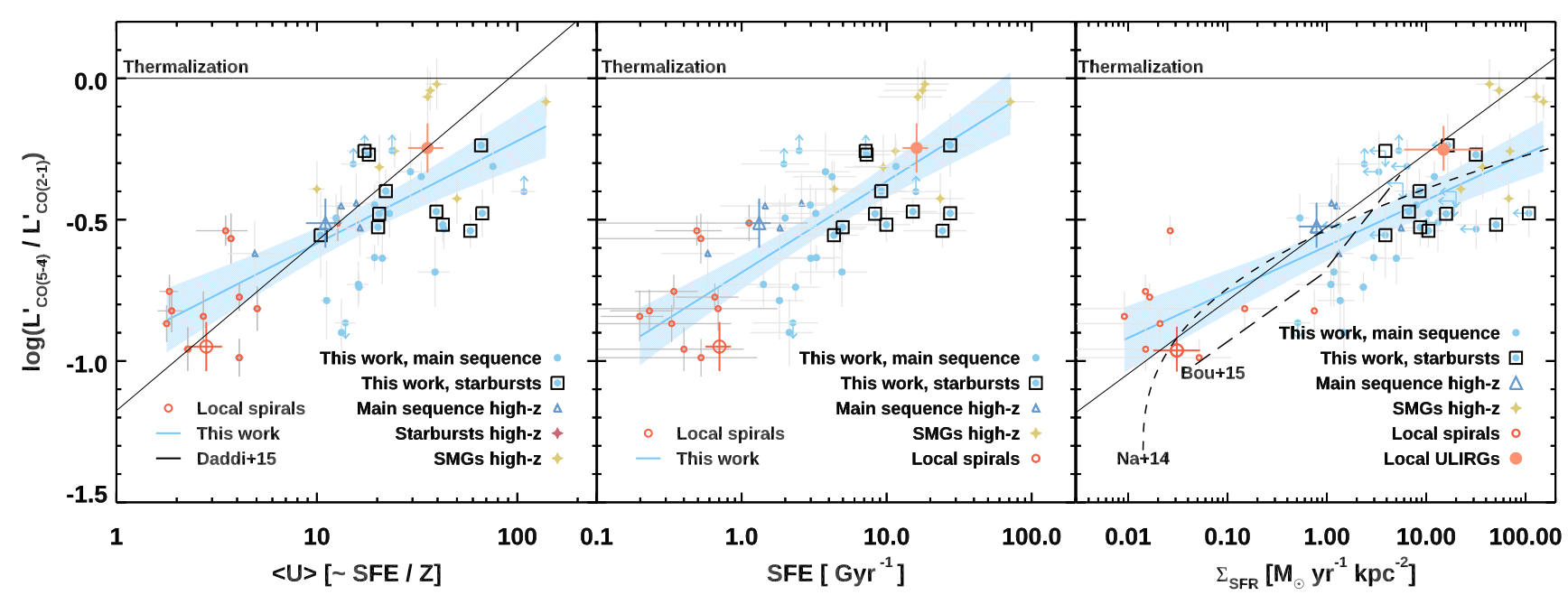

Fig. 7. Physical drivers of the CO excitation. $L^{\prime}\left[\mathrm{K} \mathrm{km} \mathrm{s}^{-1} \mathrm{pc}^{2}\right]$ luminosity ratio between $\mathrm{CO}(5-4)$ and $\mathrm{CO}(2-1)$ as a function of $\langle U\rangle$ from SED modeling (left), SFE (center), and $\Sigma_{\text {SFR }}$ (right). Symbols are color-coded as labeled (see Sect. 3.6 for references), where our samples of main-sequence and starburst galaxies at $z \sim 1.3$ are marked by blue filled circles and open black squares, respectively. The solid blue line and the shaded area mark the best-fit model from the linear regression analysis and its $95 \%$ confidence interval (see Table 3 for the parameters). The solid black line shows the best-fit model from D15, based on the average values for local spirals, ULIRGs, and $B z K \mathrm{~s}$ at $z=1.5$ (large open red circle, filled red circle, and open blue triangle, respectively). The short- and long-dashed black lines in the right panel indicate the predicted trends from the simulations by Narayanan \& Krumholz (2014) and Bournaud et al. (2015), respectively.

measurements in Liu et al. (2015b) for the local spirals. For reference, we also show the mean values for the $B z K$ galaxies, the local spirals, and ULIRGs as in D15. The best-fit model to the observed points returns a $60 \%$ flatter slope than in D15 (Table 3), but the trends are qualitatively similar. We restate that the choice of the tracer, the resolution, and depth of the observations play a major role in setting the exact values of the slope and intercept in our simple linear model, which should be thus taken with a grain of salt. This is particularly true for spatially resolved local objects, where we attempted to replicate the global, galaxy-scale measurements that can be obtained for distant objects. The observed data points in Fig. 7 qualitatively agree with the simulations by Narayanan \& Krumholz (2014) and Bournaud et al. (2015), and they support the validity of $\Sigma_{\mathrm{SFR}}$ as a good proxy for the gas conditions in galaxies. The total SFR is a worse predictor of the gas excitation conditions ( $\mathrm{Lu}$ et al. 2014; Kamenetzky et al. 2016), since it does not correlate with the density and temperature probability distribution functions in clouds (Narayanan \& Krumholz 2014). Interestingly, this seems to be partially confirmed by the linear regression analysis we applied here (Table 3): when modeling $R_{52}$ as a function of $L_{\mathrm{IR}}$ $\left(\propto\right.$ SFR, Fig. 5) and $\Sigma_{\mathrm{SFR}}$, we do find similar slopes, but also a larger correlation coefficient $\rho$ for $\Sigma_{\mathrm{SFR}}$ than for $L_{\mathrm{IR}}$. However, $L_{\mathrm{IR}}$ alone does correlate with the $\mathrm{CO}$ line luminosity ratio.

\subsection{The CO spectral line energy distribution of distant main-sequence galaxies}

Given the large number of galaxies with available and reliable information on $\mathrm{CO}(2-1)$ and $\mathrm{CO}(5-4)$, in the previous sections we used the ratio of these two lines as a proxy for the $\mathrm{CO}$ excitation. However, information of $\mathrm{CO}(4-3)$ and $\mathrm{CO}(7-6)$ is now available for a subsample of distant main-sequence galaxies, which can be used to constrain their full CO SLED. In Fig. 8 we show the average SLEDs for main-sequence and starbursting sources and we compare them with a selection from the literature representative of several different galaxy populations. The latter range from the inner disk of the Milky Way (Fixsen et al.
1999) and local ULIRGs (Papadopoulos et al. 2012; see also Lu et al. 2014 and Kamenetzky et al. 2016 for extended libraries of local IR-bright objects), to $B z K$-selected star-forming objects (D15), variously selected high-redshift SMGs (Bothwell et al. 2013; Spilker et al. 2014), and QSOs (Carilli \& Walter 2013). The mean and median $L^{\prime}$ luminosities for our new SLEDs of main-sequence and starburst galaxies, along with their uncertainties, are reported in Table 4 . We computed these values for the objects meeting the requirements for a potential $\mathrm{CO}(2-1)$ follow-up, based on the updated IR photometry. These galaxies largely overlap with the sample that was effectively observed and reliably characterized (Appendix B) and restraining the analysis to the latter does not affect the results of the following sections, while extending the calculation to all the galaxies with $\mathrm{CO}(5-4)$ would artificially decrease the observed ratios. The imposed condition further implies similar $L_{\mathrm{IR}}$ for the galaxies entering the analysis. We note that the median $L_{\mathrm{IR}}$ for the mainsequence objects with $\mathrm{CO}(4-3)$ and $[\mathrm{CI}]\left({ }^{3} P_{1}-{ }^{3} P_{0}\right)$ coverage is 0.2 dex smaller than the median value of the galaxies that we consider for the remaining transitions. This might imply an underestimate of the $L^{\prime}{ }_{\mathrm{CO}(4-3)}$ and $\left.L^{\prime}{ }_{[\mathrm{C}]}\right]^{3} P_{1}-{ }^{3} P_{0}$ luminosities by the same factor, for constant $L^{\prime} / L_{\mathrm{IR}}$ ratios. However, this difference is well within the observed range of ratios (V20) and it does not affect the essence of the results presented in the coming sections. As in the previous sections, we did not include AGNdominated objects $\left(f_{\mathrm{AGN}} \geq 80 \%\right.$ of the total $L_{\mathrm{IR}}$, Sect. 3.4) in the analysis. A study of their SLEDs and the contribution of Xray dominated regions (XDR) is postponed to future work. Nevertheless, we remark that the distribution of $f_{\mathrm{AGN}}$ is consistent between the samples of main-sequence and the starburst objects analyzed in this Section. For reference and completeness, we show the SLEDs for the subsample with detected CO (2-1) and $\mathrm{CO}(5-4)$ in Fig. C.1. We show the SLEDs in terms of fluxes to facilitate the comparison with D15, where we normalized all the curves to the mean $\mathrm{CO}(2-1)$ line flux of our main-sequence sample. The fluxes are computed at the median redshift of our sample $(z=1.25)$, after averaging the luminosities to remove the distance effect. 

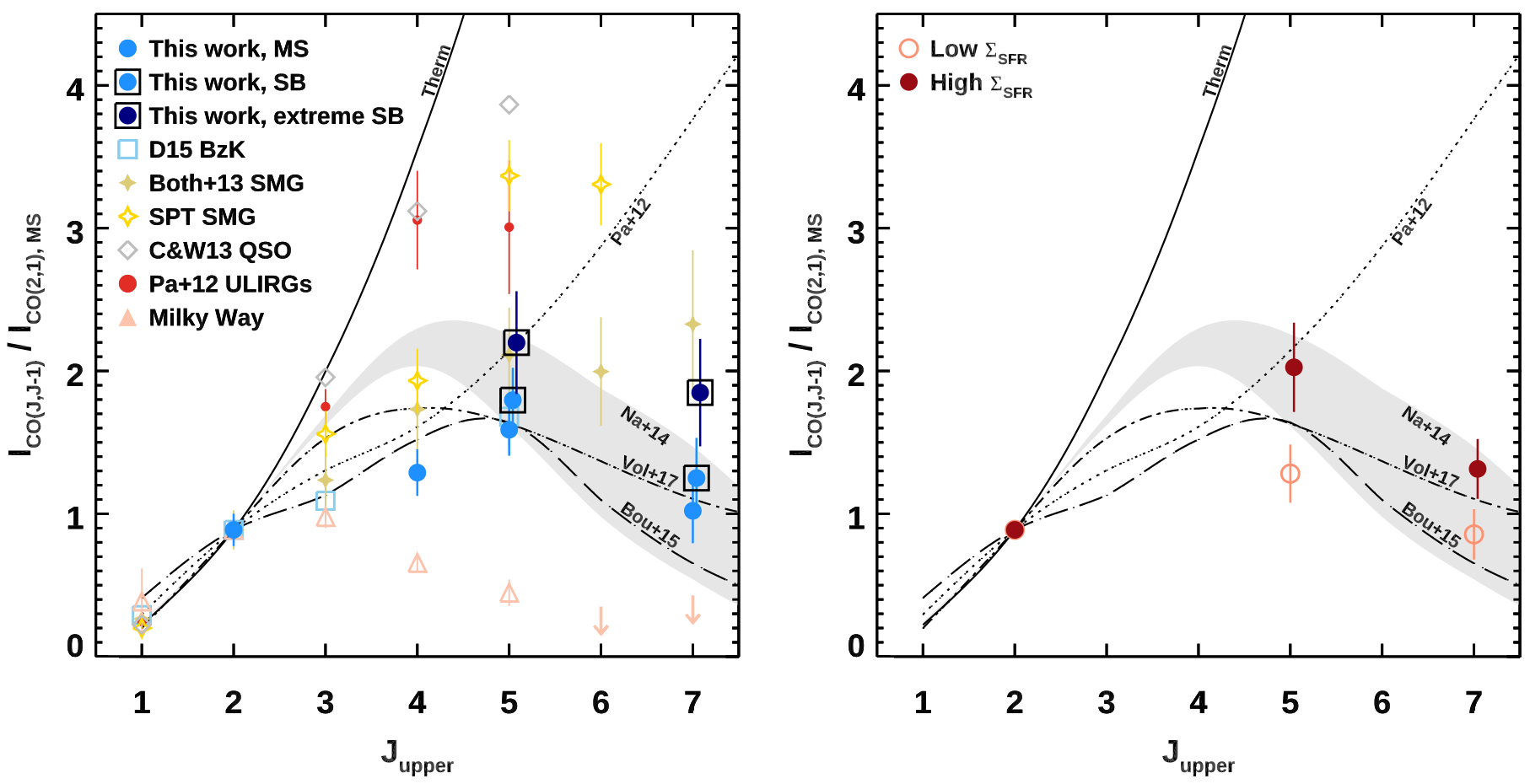

Fig. 8. CO excitation ladder. Left: average CO SLEDs of our samples compared with results from the literature. Each SLED is normalized to the mean $\mathrm{CO}(2-1)$ flux of main-sequence galaxies at $z=1.25$. Blue filled circles and open black squares indicate the mean SLEDs of galaxies on the main sequence, starbursts ( $\triangle \mathrm{MS} \geq 3.5$ ), and extreme starbursts ( $\triangle \mathrm{MS} \geq 7$ ), as labeled. The literature samples include the $B z K$ galaxies from D15 (open light blue squares); the average SMGs from Bothwell et al. (2013) (filled green stars) and the SPT-selected ones from Spilker et al. (2014) (open golden stars); high-redshift QSOs from Carilli \& Walter (2013) (open gray diamonds); local ULIRGs from Papadopoulos et al. (2012) (red filled circles); the inner disk of the Milky Way from Fixsen et al. (1999) (open pink triangles). The upper limits are at 3 $\sigma$ significance. The solid line shows the line ratios for a fully thermalized case. The gray shaded area marks the model by Narayanan \& Krumholz (2014) for unresolved observations within $\Sigma_{\mathrm{SFR}}=1-10 M_{\odot} \mathrm{yr}^{-1} \mathrm{kpc}^{-2}$. The long dashed line indicates the simulations from Bournaud et al. (2015). The dotted line traces the empirical model by Papadopoulos et al. (2012). The dashed-dotted line points at the analytical model by Vollmer et al. (2017). Right: mean CO SLEDs for a subsample of objects with detections of $\mathrm{CO}(2-1), \mathrm{CO}(5-4)$, and estimates of $L_{\mathrm{IR}}$ and FIR sizes, split at the median value of $\Sigma_{\mathrm{SFR}}$. Light empty and dark filled red circles indicate the low and high $\Sigma_{\mathrm{SFR}}$ subsamples. Both SLEDs are normalized to the CO (2-1) flux of the main-sequence sample as in the left panel.

\subsubsection{CO SLEDs across different galaxy populations}

The average SLED for main-sequence galaxies at $z \sim 1.25$ appears significantly more excited than the inner disk of the Milky Way, but not as excited as local ULIRGs or highredshift SMGs and QSOs. Predictably, it is also substantially subthermally excited already at mid- $J$ transitions (Dannerbauer et al. 2009). On average, the $R_{52}=L_{\mathrm{CO}(5-4)}^{\prime} / L_{\mathrm{CO}(2-1)}^{\prime}=$ $I_{\mathrm{CO}(5-4)} / I_{\mathrm{CO}(2-1)} /(J=5 / J=2)^{2}$ ratio for galaxies on the main sequence (Table 5) is $1.8 \times$ smaller than ULIRGs (Papadopoulos et al. 2012), 1.3 $\times$ and 2.1× than SMGs from Bothwell et al. (2013) and Spilker et al. (2014), and $2.4 \times$ than distant QSOs (Carilli \& Walter 2013). However, the $R_{52}$ ratio is 3.6x higher than the observed values in the disk of the Milky Way (Fixsen et al. 1999). Similar considerations apply for $\mathrm{CO}(7-6)$.

\subsubsection{CO SLEDs on and above the main sequence}

The excitation of the average SLEDs only tentatively increases with the distance from the main sequence. At mid- and high$J$ transitions, the $L_{J}^{\prime} / L_{\mathrm{CO}(2-1)}^{\prime}$ ratios are $1.1 \times$ and $1.2 \times$ higher for starbursts than main-sequence galaxies for $J=5$ and 7 , respectively (Table 5). This difference and its low significance depend on the threshold for the definition of starbursts, currently set at $\Delta \mathrm{MS} \geq 3.5$; the averaging of all galaxies in only two bins of $\triangle \mathrm{MS}$, further softening the trend shown in Fig. 6; and an intrinsic diversity of shapes of CO SLEDs even within a sample of homogeneously selected galaxies (Fig. C.1). We note that the latter strongly affects any estimate of the molecular gas mass from excited $\mathrm{CO}$ transitions. A more extreme threshold for the starburst regime results in an increase of the deviation between the two samples and of its significance. The difference in $L_{J}^{\prime} / L_{\mathrm{CO}(2-1)}^{\prime}$ ratios rises to $1.4 \times, 1.8 \times$ at $J=5,7$ for $\Delta \mathrm{MS} \geq 7$, substantially increasing the CO fluxes for the starbursts and bringing them closer to the typical values of SMGs (Fig. 8). The shape also looks flatter, similarly to local IR-bright galaxies (Mashian et al. 2015; Kamenetzky et al. 2016). However, this happens at the expense of the number statistics, which are too sparse for a definitive conclusion about such an extreme definition of starbursts.

The average $R_{52}$ ratio for the IR-selected main-sequence objects is similar to the previous estimate for the four $B z K$ selected galaxies from D15. We note that the addition of the $J=7$ transition constrains the peak of the main-sequence SLED to lower $J$, showing a significant departure from other more extreme populations of galaxies. However, the overall shape of the SLED is flatter than the rapid decrease observed in the disk of the Milky Way, suggesting the existence of a secondary warm component and excluding a steady increase at every $J$ observed so far. 
Table 4. Average emission line luminosities for galaxies at $z \sim 1.25$.

\begin{tabular}{|c|c|c|c|}
\hline \multicolumn{4}{|c|}{ Main sequence } \\
\hline Transition & $N_{\text {det }}, N_{\text {up }}$ & Mean & Median $^{(a)}$ \\
\hline$L_{\mathrm{CO}(2-1)}^{\prime}$ & 18,4 & $1.83 \pm 0.23^{(\dagger)}$ & $1.62_{-0.70}^{+0.30}$ \\
\hline$L_{\mathrm{CO}(4-3)}^{\prime}$ & 4,0 & $0.66 \pm 0.08$ & $0.71_{-0.12}^{+0.16}$ \\
\hline$L_{\mathrm{CO}(5-4)}^{\prime}$ & 20,2 & $0.52 \pm 0.06^{(\dagger)}$ & $0.44_{-0.11}^{+0.26}$ \\
\hline$L_{\mathrm{CO}(7-6)}^{\prime}$ & 6,0 & $0.17 \pm 0.04$ & $0.17_{-0.06}^{+0.01}$ \\
\hline$L_{[\mathrm{C} I]^{3} P_{1}-{ }^{3} P_{0}}^{\prime}$ & 7,1 & $0.37 \pm 0.05^{(\dagger)}$ & $0.31_{-0.07}^{+0.09}$ \\
\hline$L_{\left[\mathrm{CI}_{\mathrm{I}}\right]^{3} P_{2}-{ }^{3} P_{1}}$ & 6,0 & $0.17 \pm 0.03$ & $0.19_{-0.11}^{+0.04}$ \\
\hline \multicolumn{4}{|c|}{ Starbursts } \\
\hline Transition & $N_{\mathrm{det}}, N_{\text {up }}$ & Mean & Median $^{(a)}$ \\
\hline$L_{\mathrm{CO}(2-1)}^{\prime}$ & 11,1 & $1.91 \pm 0.24(\dagger)$ & $1.90_{-1.06}^{+0.38}$ \\
\hline$L_{\mathrm{CO}(4-3)}^{\prime}$ & - & - & - \\
\hline$L_{\mathrm{CO}(5-4)}^{\prime}$ & 15,0 & $0.62 \pm 0.08$ & $0.50_{-0.10}^{+0.20}$ \\
\hline$L_{\mathrm{CO}(7-6)}^{\prime}$ & 6,0 & $0.22 \pm 0.05$ & $0.22_{-0.10}^{+0.06}$ \\
\hline 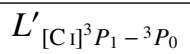 & - & - & - \\
\hline$L_{\left[\mathrm{C}_{1}\right]^{3} P_{2}-{ }^{3} P_{1}}^{\prime}$ & 6,0 & $0.20 \pm 0.04$ & $0.19_{-0.08}^{+0.05}$ \\
\hline \multicolumn{4}{|c|}{ Extreme starbursts } \\
\hline Transition & $N_{\text {det }}, N_{\text {up }}$ & Mean & Median $^{(a)}$ \\
\hline$L_{\mathrm{CO}(2-1)}^{\prime}$ & 6,1 & $1.80 \pm 0.27^{(\dagger)}$ & $1.87_{-1.05}^{+0.28}$ \\
\hline$L_{\mathrm{CO}(4-3)}^{\prime}$ & - & - & - \\
\hline$L_{\mathrm{CO}(5-4)}^{\prime}$ & 6,0 & $0.72 \pm 0.12$ & $0.58_{-0.13}^{+0.22}$ \\
\hline$L_{\mathrm{CO}(7-6)}^{\prime}$ & 3,0 & $0.31 \pm 0.06$ & - \\
\hline$L_{\left[\mathrm{C}_{1}\right]^{3} P_{1}-{ }^{3} P_{0}}$ & - & - & - \\
\hline$L_{\left[\mathrm{CI}^{\prime}\right]^{3} P_{2}-{ }^{3} P_{1}}$ & 3,0 & $0.27 \pm 0.06$ & - \\
\hline
\end{tabular}

Notes. The $L^{\prime}$ luminosities are expressed in $10^{10} \mathrm{~K} \mathrm{~km} \mathrm{~s}^{-1} \mathrm{pc}^{2}$. The average $I$ fluxes in Jy km s${ }^{-1}$ shown in Figs. 8 and 9 are computed adopting $z=1.25$. The main sequence is parameterized as in Sargent et al. (2014). Galaxies are defined as starbursts if $\Delta \mathrm{MS} \geq 3.5$ and extreme starbursts if $\Delta \mathrm{MS} \geq 7$. ${ }^{(\dagger)}$ Formally biased mean value, as the first upper limit was turned into a detection for the calculation of the KM estimator (Kaplan \& Meier 1958). ${ }^{(a)}$ The uncertainty is the interquartile range.

Table 5. Average line luminosities ratios for galaxies at $z \sim 1.25$.

\begin{tabular}{lccc}
\hline \hline Transition & Main sequence & Starbursts & Extreme starbursts \\
\hline$R_{42}$ & $0.36 \pm 0.06$ & - & - \\
$R_{52}$ & $0.28 \pm 0.05$ & $0.32 \pm 0.06$ & $0.40 \pm 0.09$ \\
$R_{72}$ & $0.09 \pm 0.02$ & $0.12 \pm 0.03$ & $0.17 \pm 0.04$ \\
$R_{[\mathrm{CI}]}$ & $0.46 \pm 0.10$ & - & - \\
\hline
\end{tabular}

Notes. The ratios and their uncertainties are computed analytically based on the mean $L^{\prime}$ luminosities in Table 4. Starbursts and extreme starbursts are defined as lying $\Delta \mathrm{MS} \geq 3.5$ and $\geq 7$ above the main sequence, respectively. $R_{42}=L_{\mathrm{CO}(4-3)}^{\prime} / L_{\mathrm{CO}(2-1)}^{\prime} ; R_{52}=L_{\mathrm{CO}(5-4)}^{\prime} / L_{\mathrm{CO}(2-1)}^{\prime}$; $R_{72}=L_{\mathrm{CO}(7-6)}^{\prime} / L_{\mathrm{CO}(2-1)}^{\prime} ; R_{[\mathrm{CI}]}=L_{[\mathrm{CI}](2-1)}^{\prime} / L_{[\mathrm{C}](1-0)}^{\prime}$.

\subsubsection{Modeling of the CO SLEDs}

The observed SLEDs allow for an assessment of the physically motivated predictions from models and simulations. To simplify the comparison with previous work, in Fig. 8 we show the same tracks reported in D15: the empirical model from Papadopoulos et al. (2012) and the hydrodynamical simula- tions from Narayanan \& Krumholz (2014) and Bournaud et al. (2015). Papadopoulos et al. (2012) assumes a hypothetical gasrich disk with a $10 \%$ of the molecular gas in a star-forming phase with Orion A/B-like excitation, along with a quiescent component with an excitation as in the Milky Way. Narayanan \& Krumholz (2014) applied a radiative transfer code to simulated disks and mergers to calculate CO SLEDs as a function of $\Sigma_{\text {SFR }}$. We adopted their prescription for unresolved observations for galaxies with $\Sigma_{\mathrm{SFR}}=1-10 M_{\odot} \mathrm{yr}^{-1} \mathrm{kpc}^{-2}$, which are typical for our main-sequence and starburst galaxies (Fig. 7). Bournaud et al. (2015) applied a large velocity gradient model to high-resolution simulations and computed a synthetic $\mathrm{CO}$ emission. In particular, they distinguish the high excitation of dense clumpy medium with the less extreme conditions of the diffuse gas. Here we add the analytical model for high-redshift starforming galaxies by Vollmer et al. (2017). The simulations and the analytical model qualitatively reproduce the rise of the $\mathrm{CO}$ SLED of main-sequence objects until mid- $J$ transitions and the following smooth decrease, while the constant rising predicted by Papadopoulos et al. (2012) does not appear to be followed by the average observations. The model by Vollmer et al. (2017) appears to best catch the flat shape at high- $J$, while the simulations from Bournaud et al. (2015) describe well the location of the peak for our samples. The full treatment of the gas physics in simulations and the analytical model seems to capture the main features of the SLEDs of our sample, even if the exact shape and the flux normalizations are partially inconsistent with the observations. However, we warn the reader that the shape of average observed SLEDs is influenced by a mix of galaxies covering a range of excitation conditions, while the modeled profiles are typical of each individual object (at least for the simulations of Bournaud et al. 2015). For a definitive assessment of the various models, it will be critical to extend the coverage to higher$J$ emission, where their predictions are mostly diverging (see Kamenetzky et al. 2016 and Vollmer et al. 2017 for a discussion about the performances for ULIRGs and SMGs at $J>6$ ).

\subsubsection{Large velocity gradient modeling}

Large Velocity Gradient (LVG) modeling is a classical approach to gain insight into the properties of the molecular gas in galaxies (Goldreich \& Kwan 1974; Scoville \& Solomon 1974; Young \& Scoville 1991; Papadopoulos \& Seaquist 1998). Here we followed the approach used in D15 (Liu et al., in prep.). First, we used the RADEX tool (van der Tak et al. 2007) to create a grid of LVG models. We adopted the collisional rates from Flower (2001) with an ortho-to-para ratio of 3, and a CO abundance to velocity gradient ratio of $\left[\mathrm{CO} / \mathrm{H}_{2}\right] /(\mathrm{d} v / \mathrm{d} r)=10^{-5} \mathrm{~km} \mathrm{~s}^{-1} \mathrm{pc}^{-1}$ valid for solar metallicities (Weiß et al. 2005, 2007). We computed a model grid for the median redshift of the sample $(z=$ $1.25)$, covering density and temperature intervals of $n\left(\mathrm{H}_{2}\right)=$ $10^{2}-10^{6} \mathrm{~cm}^{-3}$ and $T_{\text {kin }}=5-300 \mathrm{~K}$, including the appropriate value of the temperature of the cosmic microwave background. We fixed the line width to $50 \mathrm{~km} \mathrm{~s}^{-1}$ or, equivalently, the cloud scale height to $10 \mathrm{pc}$, values typical of giant molecular clouds (GMCs). Given the limited amount of information, leaving these parameters or $\mathrm{d} v / \mathrm{d} r$ free to vary would result in overfitting. Considering that a galaxy contains many of these LVG clouds, the beam filling factor is simply their number. We caution the reader that due to the high degeneracy among the LVG parameters, even with a handful of $\mathrm{CO}$ lines one could not obtain 


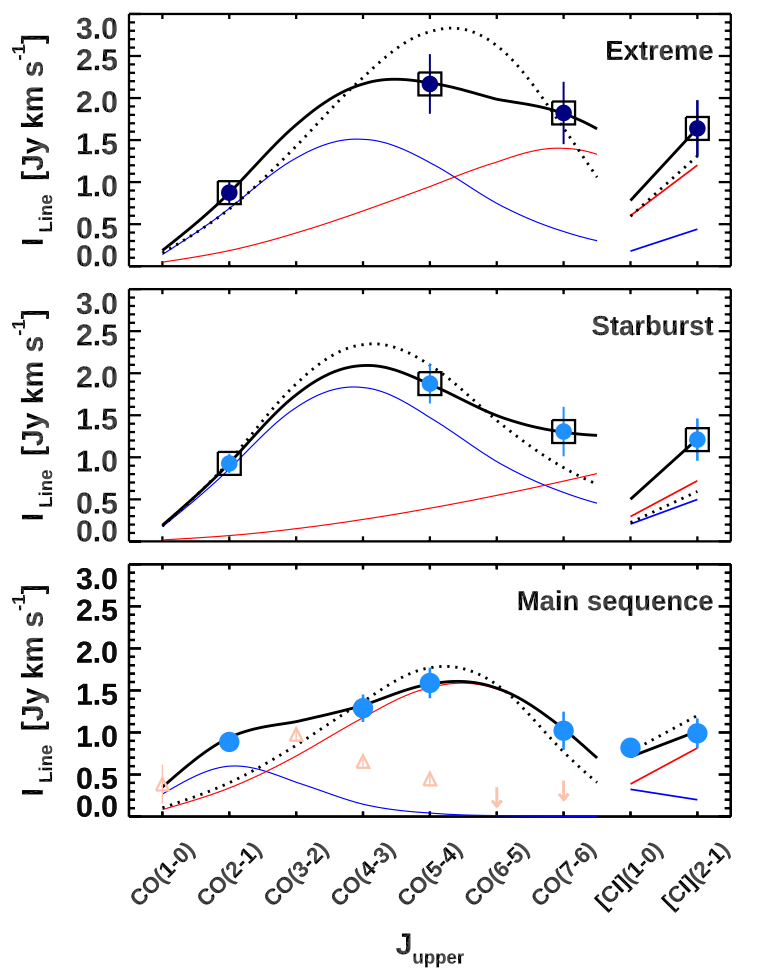

Fig. 9. LVG modeling. Large velocity gradient modeling of the observed $\mathrm{CO}+[\mathrm{CI}]$ SLEDs for main-sequence (bottom panel), starburst ( $\triangle \mathrm{MS} \geq 3.5$, central panel), and extreme starburst galaxies ( $\Delta \mathrm{MS} \geq 7$, top panel) from our ALMA survey. The filled symbols show the mean fluxes. The blue and red lines show the low- and high-excitation components of the modeling, with the black solid line indicating their sum. The dotted black line shows the best-fit model with a single component. For reference, we show the CO data for the inner disk of the Milky Way (Fixsen et al. 1999) normalized to the CO $(2-1)$ emission of the average main-sequence galaxies as in Fig. 8 (pink open triangles). The upper limits are at $3 \sigma$ significance.

results simultaneously constraining $n_{\mathrm{H}_{2}}, T_{\text {kin }}$, and $N\left(\mathrm{H}_{2}\right)$. Nevertheless, we verified that our estimates of $N\left(\mathrm{H}_{2}\right)$ and the line optical depths are within the reasonable physical ranges for GMCs (Glover et al. 2015; Tress et al. 2020). All things considered, the best-fit $n_{\mathrm{H}_{2}}$ and $T_{\text {kin }}$ in this work mostly reflect the relative trends between the different subsamples of main-sequence and starburst galaxies.

We determined the best-fit model via a customized $\chi^{2}$ minimization algorithm optimized for the exploration of highly multidimensional spaces (MICHI ${ }^{4}$, Liu 2020). In particular, we iteratively sampled the $\chi^{2}$ distribution 15000 times, randomizing the parameters within normal distributions centered on the lowest $\chi^{2}$ derived at the previous iteration, but artificially inflating their width. The output consists in best-fit (i.e., $\min \left(\chi^{2}\right)$ ) parameters and their $\sigma$ uncertainties, plus median and $68 \%$ interpercentile values for an arbitrarily large number of components. To better constrain the fit, we further included the [C I] transitions under the assumption that the neutral atomic carbon is cospatial with $\mathrm{CO}$. We generated the models with RADEX assuming a fixed abundance of $[\mathrm{CI}] / \mathrm{H}_{2}=3 \times 10^{-5}$ (Weiß et al. 2003; Papadopoulos et al. 2004, but see V18 on the reliability of this assumption). We independently fit the line fluxes for the average main-sequence and starburst samples (Table 4).

It is evident from Fig. 9 that single components do not provide a good representation to the observed CO SLEDs of both

\footnotetext{
4 https://ascl.net/code/v/2533
}

Table 6. Best-fit parameters of a double-component LVG modeling of the observed $\mathrm{CO}+[\mathrm{CI}]$ SLEDs of galaxies at $z \sim 1.25$.

\begin{tabular}{|c|c|c|}
\hline \multicolumn{3}{|c|}{ Main sequence } \\
\hline & Low & High \\
\hline $\log \left(n_{\mathrm{H}_{2}} /\left[\mathrm{cm}^{-3}\right]\right)$ & $2.2 \pm 0.3$ & $3.9 \pm 0.1$ \\
\hline$T_{\text {kin }} /[\mathrm{K}]$ & $45 \pm 113$ & $45 \pm 5$ \\
\hline \multicolumn{3}{|c|}{ Starburst } \\
\hline & Low & High \\
\hline $\log \left(n_{\mathrm{H}_{2}} /\left[\mathrm{cm}^{-3}\right]\right)$ & $2.9 \pm 0.8$ & $6.1 \pm 1.4$ \\
\hline$T_{\text {kin }} /[\mathrm{K}]$ & $300 \pm 138$ & $75 \pm 138$ \\
\hline \multicolumn{3}{|c|}{ Extreme starburst } \\
\hline & Low & High \\
\hline $\log \left(n_{\mathrm{H}_{2}} /\left[\mathrm{cm}^{-3}\right]\right)$ & $3.0 \pm 0.8$ & $4.5 \pm 1.3$ \\
\hline$T_{\text {kin }} /[\mathrm{K}]$ & $215 \pm 138$ & $35 \pm 138$ \\
\hline
\end{tabular}

Notes. The average values and their uncertainties are the best-fit estimates and their statistical errors, where we imposed that $n_{\mathrm{H}_{2} \text {, low }}<$ $n_{\mathrm{H}_{2} \text {, high }}$.

populations. This is was already suggested for $B z K$ galaxies (D15, see also Brisbin et al. 2019), and it is a well known fact for local IR-bright galaxies (Papadopoulos et al. 2010b, 2012; Lu et al. 2014; Rosenberg et al. 2015; Kamenetzky et al. 2016) and high-redshift SMGs and QSOs (e.g., Weiß et al. 2007; Aravena et al. 2008; Ivison et al. 2011; Bothwell et al. 2013; Carilli \& Walter 2013; Greve et al. 2014; Spilker et al. 2014; Yang et al. 2017; Cañameras et al. 2018). This result still holds when modeling only the $\mathrm{CO}$ emission, excluding the [C I] transitions.

The addition of a second component outperforms the previous attempt. We assumed the existence of a dense and a diffuse

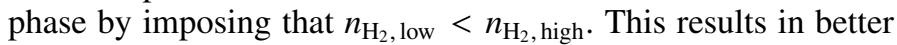
constrained densities, but not $T_{\text {kin }}$ (Table 6). This is particularly evident for the starbursts, likely due to the lack of mid- $J$ coverage. For both main-sequence and starburst galaxies we retrieve the existence of similar low- $\left(n_{\mathrm{H}_{2} \text {, low }} \sim 10^{2}-10^{3} \mathrm{~cm}^{-3}\right)$ and highdensity components $\left(n_{\mathrm{H}_{2}}\right.$, high $\left.\sim 10^{4}-10^{6} \mathrm{~cm}^{-3}\right)$. Furthermore, we retrieve a substantial amount of gas in the dense phase. The latter encloses $\sim 50 \%$ of the total molecular gas mass for mainsequence galaxies. The fraction of denser gas in starbursts is hardly constrained at this stage, possibly due to the absence of lines at $J>7$, where a substantial emission from the excited component might be expected, as shown in local ULIRGs (e.g., Mashian et al. 2015; Kamenetzky et al. 2016) and distant SMGs (e.g., Yang et al. 2017; Cañameras et al. 2018). This is suggested by the flatter shape of the SLEDs for the more extreme starburst with $\Delta \mathrm{MS} \geq 7$. We note that the absolute values of the gas mass from this modeling depend on the adopted $\mathrm{CO}$ abundance, constant for both main-sequence and starburst galaxies. Therefore, they are subject to the uncertainties already described in Sect. 4.3.1. Relative comparisons between the two phases for each population still hold, under the assumption that dense and diffuse gas reservoirs share the same metallicity.

\subsubsection{The effect of dust opacity on the high- $J \mathrm{CO}$ emission}

Large dust optical depths even at submillimeter wavelengths are responsible for the apparent depressed high- $J \mathrm{CO}$ emission in extreme objects such as Arp 220 (Greve et al. 2009; Papadopoulos et al. 2010a; Rangwala et al. 2011; Scoville et al. 2017b). This is due to the fact that for $\tau_{\text {dust }} \gg 1$, the line emissions are largely 
erased by the quasi-black body dust continuum (Papadopoulos et al. 2010a). A modeling of the dust continuum emission leaving the optical depth free to vary can provide meaningful results only when the SED is well sampled from mid-IR to millimeter wavelengths and it might still return degenerate solutions with the temperature $T_{\text {dust }}$, in absence of independent ways to distinguish them (Cortzen et al. 2020). Indeed, our SED modeling is based on the assumption that the dust emission is optically thin above $100 \mu \mathrm{m}$. Here we tested our assumption by computing the dust optical depth at the $\mathrm{CO}(5-4)$ and $\mathrm{CO}(7-6)$ wavelengths as $\tau_{\text {dust }}=\kappa(v) \Sigma_{d}=\kappa_{850 \mu \mathrm{m}}\left(v / v_{850 \mu \mathrm{m}}\right)^{\beta} M_{\text {dust }} /\left(2 \pi R^{2}\right)$, where $\kappa$ is the frequency-dependent dust opacity, and $\Sigma_{d}$ the dust mass surface density (e.g., Casey et al. 2014). We adopted $\kappa_{850 \mu \mathrm{m}}=$ $0.43 \mathrm{~cm}^{-2} \mathrm{~g}^{-1}$ and $\beta=2$ (Li \& Draine 2001), the $M_{\text {dust }}$ from the SED modeling (Sect. 3.3), and the sizes from the ALMA measurements. We note that, while using one of the outputs of the SED fitting, this sanity check is not tautological, given the introduction of the size in the calculation. For sources with a significant $\mathrm{CO}(5-4)$ line detection, a determination of $M_{\text {dust }}$ and of the size from ALMA, we retrieve $\left\langle\tau_{\text {dust }}(520 \mu \mathrm{m})_{\mathrm{CO}(5-4)}\right\rangle=0.012 \pm 0.003$, including the lower limits on $\tau_{\text {dust }}$ due to the upper limits on the sizes. For the dust continuum emission under $\mathrm{CO}(7-6)$, we find $\left\langle\tau_{\text {dust }}(371 \mu \mathrm{m})_{\mathrm{CO}(7-6)}\right\rangle=0.020 \pm 0.007$, with a maximum of 0.08 , where all the $\mathrm{CO}(7-6)$ detections have a safe determination of their size. We note that the same calculation with $M_{\text {dust }}$ derived from an ideal SED modeling with the opacity as a free parameter would be lower, further decreasing the value of $\tau_{\text {dust }}$.

The largest opacities are associated with strongly starbursting galaxies or AGN contamination, but their observed high$J /$ low- $J$ CO ratios do not appear systematically depressed compared to the rest of the sample. However, they do have $\tau_{\text {dust }}=1$ at rest-frame wavelengths of $\lambda \sim 100-140 \mu \mathrm{m}$, similar to several high-redshift SMGs. This suggests that their $M_{\text {dust }}$ and $T_{\text {dust }}$ are likely over- and underestimated, respectively (Jin et al. 2019; Cortzen et al. 2020). These cases represent $<5 \%$ of the sample for which we could carry out the test on the opacity and they do not influence the final results. Therefore, it appears that the dust opacity does not have a significant impact on the emission of mid- and high- $J$ CO lines on global scales in our sample and this is due to the less extreme $\Sigma_{\text {dust }}$ compared with, for example, the value of Arp 220. However, we notice that this is a point to be reassessed with higher spatial resolution measurements, which might well reveal compact pockets of gas more affected by the dust absorption within our targets.

\section{Discussion}

In the previous sections, we showed that, on global scales, the $\mathrm{CO}$ line emission and excitation of main-sequence and starburst galaxies broadly correlate with a variety of properties. The high$J$ CO line luminosities $(J=5,7)$ are quasi-linearly related to the star formation rate, suggesting a physical connection with the gas pockets where new stars are formed. The low- $J$ CO line emission is associated with less dense molecular gas, tracing the bulk of its mass in galaxies. Interestingly, the $L_{\mathrm{CO}(5-4)}^{\prime} / L_{\mathrm{CO}(2-1)}^{\prime}$ ratio and overall CO SLED increase as a function of the total infrared luminosity $L_{\mathrm{IR}}(\propto \mathrm{SFR})$, the mean intensity of the radiation field heating the dust $\langle U\rangle$ ( $\propto T_{\text {dust }}$ ), the star formation efficiency SFE $=\operatorname{SFR} / M_{\star}$, the surface density of SFR $\left(\Sigma_{\mathrm{SFR}}\right)$, and, less distinctly, with the distance from the main sequence $\Delta \mathrm{MS}$. A comparison of the strength of the observed correlations and their intrinsic scatter (Table 3) offers further insight into this network of properties.

\subsection{The spatial distribution of SFR as the driver of the properties of star-forming galaxies}

The main physical driver of the $\mathrm{CO}$ excitation seems to be a combination of the amount of star formation occurring in the galaxy and its spatial distribution. While a SFR- $R_{52}$ correlation does exist, its strength increases by using $\Sigma_{\text {SFR }}$ instead. This naturally follows the fact that dense gas concentrations ignite more compact star-forming regions, producing large UV radiation fields and cosmic ray rates and warming up the dust (Narayanan \& Krumholz 2014). This is reflected on the similarly strong and tight $\langle U\rangle-R_{52}$ relation and on the enhancement of the excitation as a function of the SFE, boosted in more compact gas configurations (Papadopoulos et al. 2012). We note that the correlation between the $\mathrm{CO}$ excitation and $\langle U\rangle$ does not necessarily imply that the interstellar radiation field is responsible for the excitation of the mid- and high- $J$ transitions. If multicomponent photon-dominated regions (PDRs) have been shown to be sufficient to describe the CO SLED of local spirals (Rigopoulou et al. 2013), this is not the case for starbursts, where mechanical heating induced by SF-related (supernovae) or unrelated (mergers, AGN outflows, radio-jets) shocks are invoked to explain the observations (Rangwala et al. 2011; Kamenetzky et al. 2012, 2016; Lu et al. 2014; see Brisbin et al. 2019 for the case of a distant main-sequence galaxy). The correlation with $\langle U\rangle$ would then be indirect: it is the stellar feedback from the intense star formation to drive it (Wu et al. 2015). This might well be the case for the most extreme starbursts, for which PDRs cannot reproduce the observed flat CO SLEDs (Kamenetzky et al. 2016). We note that the models in Fig. 8 specifically contemplating a recipe for the mechanical heating from SNae better reproduce the flat shapes of the CO SLEDs and the location of their peaks (Bournaud et al. 2015; Vollmer et al. 2017, and see the discussion in Papadopoulos et al. 2012).

The global rise of $R_{52}$ with the distance from the main sequence can be interpreted considering that $\Sigma_{\text {SFR }}$ overall increases with $\triangle \mathrm{MS}$ (Elbaz et al. 2011). Moreover, the high gas fractions inducing clumping in turbulent high-redshift massive disks further enhance this effect (Bournaud et al. 2015), increasing the level of $\mathrm{CO}$ excitation of distant main-sequence galaxies with respect to local Milky Way-like objects. In other words, the same mechanisms that we consider here as acting on global galaxy scales might well be in action in subgalactic massive clumps, effectively mimicking starburst environments (Zanella et al. 2015, 2019). This is also consistent with the results from LVG modeling, where the density of the collisionally excited gas is the term driving the high- $J$ emission, given the fast deexcitation rates. Finally, taken to its extreme consequences, the formation of hundreds or even thousands of stars per $\mathrm{kpc}^{2}$ and the ensuing massive layers of dust surrounding them (e.g., Arp 220, Barcos-Muñoz et al. 2015; Scoville et al. 2017b) affect the emerging $\mathrm{CO}$ spectrum of a galaxy, inducing the uttermost opacities at long wavelengths (e.g., Blain et al. 2003; Greve et al. 2009; Riechers et al. 2013; Huang et al. 2014; Lutz et al. 2016; Hodge et al. 2016; Spilker et al. 2016; Simpson et al. 2017). This effect reduces the apparent CO excitation at high- $J$ (Papadopoulos et al. 2010a; Rangwala et al. 2011) and the dust temperature, affecting the dust mass estimates if not properly taken into account (Jin et al. 2019; Cortzen et al. 2020).

\subsection{The definition of starbursts}

The scenario presented above has been formulated in various flavors to individually explain several of the properties reported 
here. The main addition of this work, namely the excitation of $\mathrm{CO}$ in distant main-sequence and starburst galaxies, fits in the general picture that we sketched. The ensemble of properties and correlations that we reported here can be also used to revisit the definition of what a starburst is. A standard operational classification is based on the distance from the observed empirical $M_{\star}$-SFR correlation, the main sequence. This proved to be a useful distinction and an excellent predictor of several trends (e.g., Sargent et al. 2014), but recent results, including our present and previous analysis (Puglisi et al. 2019), show that the demarcation between starburst and main-sequence galaxies is more blurred that we previously considered. We do detect starburst-like behaviors in galaxies on the main sequence (Elbaz et al. 2018), likely linked to the existence of transitional objects (Popping et al. 2017; Barro et al. 2017b; Gómez-Guijarro et al. 2019; Puglisi et al. 2019, and in prep. to limit the references to recent works based on submillimeter observations). Such transition might well imply an imminent increase of the SFR, driving the object in the realm of starbursts (e.g., Barro et al. 2017b), or its cessation, bringing the system back onto or even below the main sequence (Gómez-Guijarro et al. 2019; Puglisi et al. 2019), with the $\mathrm{CO}$ properties potentially able to distinguish between these two scenarios. Regardless of these transitional objects, a definition of starburst based on $\Sigma_{\mathrm{SFR}}$, rather than $\Delta \mathrm{MS}$, would naturally better account for the observed molecular gas excitation properties, dust temperatures and opacities, or SFE (see also Elbaz et al. 2011; Rujopakarn et al. 2011; Jiménez-Andrade et al. 2018; Tacconi et al. 2020). As an example, in Fig. 8 we show the mean SLED of the subsample of galaxies with both CO $(2-1)$ and $\mathrm{CO}(5-4)$ coverage, split at its median $\Sigma_{\mathrm{SFR}}$. While only tentative at this stage, this suggests a trend of increasing $\mathrm{CO}$ excitation with $\Sigma_{\text {SFR }}$, consistently with Fig. 7 and what mentioned above.

In more physical terms, the new definition would trace the observed correlations back to a common origin: the accumulation of gas and formation of stars in compact configurations, following global or local dynamical changes in the galaxy structure. The latter might be due to major mergers, known to be primary drivers of starbursts activity in the local Universe (Sanders \& Mirabel 1996), or in presence of higher gas fractions, to minor mergers (Bustamante et al. 2018; Gómez-Guijarro et al. 2018) or violent disk instabilities induced by a sudden alteration of the gravitational equilibrium, particularly effective at high redshift (e.g., Bournaud et al. 2007; Ceverino et al. 2010; Dekel \& Burkert 2014). Such gas concentrations would further increase the AGN activity (Elbaz et al. 2018), spread also in our sample. Moreover, a definition based on $\Sigma_{\mathrm{SFR}}$ would allow for the classification of SMGs, normally hard to achieve because of the lack of $M_{\star}$ determinations. This could potentially sort extended massive disks (Hodge et al. 2016, 2019) and bona fide ongoing gas-rich mergers (see Casey et al. 2014 for a review of current models for the formation of dusty star-forming galaxies). We will explore the detailed properties of our sample as a function of size and compactness in a dedicated work (Puglisi et al., in prep.).

\section{Conclusions}

We presented the outcome of a multicycle ALMA survey of the $\mathrm{CO}$ emission in IR-selected galaxies on and above the main sequence at $z \sim 1.3$. We obtained new observations of lowto high- $J$ lines that we complemented with existing samples of local and distant star-forming galaxies. In detail:
1. We report new detections of $\mathrm{CO}(5-4), \mathrm{CO}(2-1)$, and $\mathrm{CO}(7-6)\left(+[\mathrm{C} \mathrm{I}]\left({ }^{3} P_{2}-{ }^{3} P_{1}\right)\right)$ for 50,33 , and 13 galaxies, respectively, corresponding to detection rates of $\sim 50-80 \%$.

2. We found that the $\mathrm{CO}(5-4)$ and $\mathrm{CO}(7-6)$ luminosities of both main-sequence and starburst galaxies follow an almost linear and tight $\left(\sigma_{\text {int }}=0.16\right.$ dex $)$ correlation with the total $L_{\mathrm{IR}}$, as previously established for local IR-bright galaxies, spirals, and distant SMGs. On the other hand, the $\mathrm{CO}(2-1)$ emission of main-sequence and starburst galaxies is consistent with the integrated SK law. This suggests that the $\mathrm{CO}(5-4)$ and $\mathrm{CO}(7-6)$ emission is associated with the reservoirs of actively star-forming gas in galaxies, while $\mathrm{CO}(2-1)$ traces the total mass of cold and less dense molecular medium. This also suggests caution when deriving the total molecular $M_{\text {gas }}$ from high- $J$ CO transitions.

3. Moreover, we found the $\mathrm{CO}(2-1) / L_{\mathrm{IR}}$ ratio to steadily decrease as a function of the distance from the main sequence $\Delta \mathrm{MS}$, while $\mathrm{CO}(5-4) / L_{\mathrm{IR}}$ and $\mathrm{CO}(7-6) / L_{\mathrm{IR}}$ remain constant. This further supports the idea that mid- and high- $J$ transitions trace the SFR, independently of their stellar mass and redshift and within the parameter space spanned by our observations.

4. We derived monotonically increasing $\mathrm{CO}(5-4) / \mathrm{CO}(2-1)$ luminosity ratios - a proxy for the $\mathrm{CO}$ excitation - as a function of increasing star formation efficiencies, mean intensities of the radiation field $\langle U\rangle$, SFR surface densities $\Sigma_{\mathrm{SFR}}$, and, less distinctly, distances from the main sequence $\Delta \mathrm{MS}$.

5. We found the overall CO SLED of distant main-sequence galaxies up to $\mathrm{CO}(7-6)$ to be more excited than the disk of the Milky Way, but less than local ULIRGs or high-redshift SMGs and QSOs. An intrinsic variety of shapes is present, as shown by the dispersion of the observed CO luminosity ratios, blurring the distinction between the SLEDs of starbursts and upper main-sequence objects. The dust opacity does not appear to significantly suppress the high- $J$ CO emission even for the most extreme objects in our sample, due to relatively low dust mass surface densities compared with galaxies such as Arp 220, the prototypical case for this matter. However, this has to be further tested with observations at higher spatial resolution.

6. We modeled the observed $\mathrm{CO}(+[\mathrm{CI}])$ SLEDs adopting the LVG method. The addition of high- $J$ CO and [C I] lines indicates the existence of a second highly excited component both for starbursts and main-sequence galaxies, similarly to what invoked to explain the SLEDs of local ULIRGs and SMGs. Imposing the existence of a dense and a diffuse component, we retrieve a substantial amount of gas in the dense phase $\left(n_{\mathrm{H}_{2} \text {, high }} \sim 10^{4}-10^{6} \mathrm{~cm}^{-3}\right)$, contributing to $\sim 50 \%$ of the total molecular gas mass for main-sequence galaxies.

7. We interpret the $\mathrm{CO}$ excitation conditions as driven by the combination of large SFRs over compact regions. Such large $\Sigma_{\text {SFR }}$ values naturally explain the high gas densities and temperatures due to increased UV radiation fields, cosmic ray heating, as well as dust and gas coupling. Higher densities also naturally induce enhanced SFEs, as canonically advocated for starbursts. An operational definition based on $\Sigma_{\mathrm{SFR}}$ rather than on the offset from the main sequence might better separate truly starbursting galaxies from secularly evolving disks.

8. Idealized simulations, analytical, and semi-empirical models qualitatively account for the increase of the $\mathrm{CO}$ excitation in distant main-sequence and starburst galaxies peaking around $J \sim 4-5$, but starting from different premises and resulting 
in shapes and normalizations partially inconsistent with the average observed trends.

Future observations covering $\mathrm{CO}$ transitions at $J>7$ and at higher spatial resolution will be the key for a definitive assessments of several issues discussed in this work and a test for models and simulations.

Acknowledgements. We acknowledge the constructive comments from the anonymous referee that improved the content and presentation of the results. We thank Jonathan Freundlich for his guidance in using the PHIBSS-2 data products. F.V. acknowledges support from the Carlsberg Foundation Research Grant CF18-0388 "Galaxies: Rise and Death". F.V. and G.E.M. acknowledge the Villum Fonden Research Grant 13160 "Gas to stars, stars to dust: tracing star formation across cosmic time" and the Cosmic Dawn Center of Excellence funded by the Danish National Research Foundation under then Grant No. 140. G.E.M. acknowledges support from the European Research Council (ERC) Consolidator Grant funding scheme (Project ConTExt, Grant No. 648179). D.L. acknowl edges funding from the European Research Council (ERC) under the European Union's Horizon 2020 research and innovation programme (grant agreement No. 694343). Este trabajo contó con el apoyo de "CONICYT+PCI+INSTITUTO MAX PLANCK DE ASTRONOMIA MPG190030”. M.A. has been supported by the grant "CONICYT+PCI+REDES 190194". H.D. acknowledges financial support from the Spanish Ministry of Science, Innovation and Universities (MICIU) under the 2014 Ramón y Cajal program RYC-2014-15686 and AYA2017-84061-P, the later one cofinanced by FEDER (European Regional Development Funds). S.J. acknowledges financial support from the Spanish Ministry of Science, Innovation and Universities (MICIU) under grant AYA201784061-P, cofinanced by FEDER (European Regional Development Funds) Y.G.'s research is supported by National Key Basic Research and Development Program of China (grant No. 2017YFA0402704), National Natural Science Foundation of China (grant Nos. 11861131007, 11420101002), and Chinese Academy of Sciences Key Research Program of Frontier Sciences (grant No. QYZDJSSW-SLH008). In this work we made use of the COSMOS master spectroscopic catalog - kept updated by Mara Salvato -, of GILDAS and STSDAS. GILDAS, the Grenoble Image and Line Data Analysis Software, is a joint effort of IRAM and the Observatoire de Grenoble. STSDAS is a product of the Space Telescope Science Institute, which is operated by AURA for NASA. Moreover, this paper makes use of the following ALMA data: ADS/JAO.ALMA, \#2019.1.01702.S, \#2018.1.00635.S, \#2016.1.01040.S $\# 2016.1 .00171$.S, and \#2015.1.00260.S. ALMA is a partnership of ESO (representing its member states), NSF (USA) and NINS (Japan), together with NRC (Canada), MOST and ASIAA (Taiwan), and KASI (Republic of Korea), in cooperation with the Republic of Chile. The Joint ALMA Observatory is operated by ESO, AUI/NRAO, and NAOJ

\section{References}

Alaghband-Zadeh, S., Chapman, S. C., Swinbank, A. M., et al. 2013, MNRAS, 435,1493

Andreani, P., Retana-Montenegro, E., Zhang, Z.-Y., et al. 2018, A\&A, 615, A142 Aravena, M., Bertoldi, F., Schinnerer, E., et al. 2008, A\&A, 491, 173

Aravena, M., Carilli, C., Daddi, E., et al. 2010, ApJ, 718, 177

Aravena, M., Hodge, J. A., Wagg, J., et al. 2014, MNRAS, 442, 558 Aravena, M., Spilker, J. S., Bethermin, M., et al. 2016, MNRAS, 457, 4406 Aretxaga, I., Wilson, G. W., Aguilar, E., et al. 2011, MNRAS, 415, 3831 Armus, L., Mazzarella, J. M., Evans, A. S., et al. 2009, PASP, 121, 559 Barcos-Muñoz, L., Leroy, A. K., Evans, A. S., et al. 2015, ApJ, 799, 10 Barro, G., Faber, S. M., Koo, D. C., et al. 2017a, ApJ, 840, 47 Barro, G., Kriek, M., Pérez-González, P. G., et al. 2017b, ApJ, 851, L40 Bertoldi, F., Carilli, C., Aravena, M., et al. 2007, ApJS, 172, 132 Bigiel, F., Leroy, A., Walter, F., et al. 2008, AJ, 136, 2846 Blain, A. W., Barnard, V. E., \& Chapman, S. C. 2003, MNRAS, 338, 733 Bolatto, A. D., Wolfire, M., \& Leroy, A. K. 2013, ARA\&A, 51, 207 Bothwell, M. S., Smail, I., Chapman, S. C., et al. 2013, MNRAS, 429, 3047 Bothwell, M. S., Aguirre, J. E., Aravena, M., et al. 2017, MNRAS, 466, 2825 Bournaud, F., Elmegreen, B. G., \& Elmegreen, D. M. 2007, ApJ, 670, 237 Bournaud, F., Elmegreen, B. G., Teyssier, R., Block, D. L., \& Puerari, I. 2010, MNRAS, 409, 1088

Bournaud, F., Daddi, E., Weiß, A., et al. 2015, A\&A, 575, A56

Bourne, N., Dunlop, J. S., Simpson, J. M., et al. 2019, MNRAS, 482, 3135

Brinchmann, J., Charlot, S., White, S. D. M., et al. 2004, MNRAS, 351, 1151

Brisbin, D., Miettinen, O., Aravena, M., et al. 2017, A\&A, 608, A15

Brisbin, D., Aravena, M., Daddi, E., et al. 2019, A\&A, 628, A104

Bustamante, S., Sparre, M., Springel, V., \& Grand, R. J. J. 2018, MNRAS, 479 3381
Cañameras, R., Yang, C., Nesvadba, N. P. H., et al. 2018, A\&A, 620, A61 Capak, P. L., Carilli, C., Jones, G., et al. 2015, Nature, 522, 455 Carilli, C. L., \& Walter, F. 2013, ARA\&A, 51, 105 Casey, C. M., Narayanan, D., \& Cooray, A. 2014, Phys. Rep., 541, 45 Cassata, P., Liu, D., Groves, B., et al. 2020, ApJ, 891, 83 Ceverino, D., Dekel, A., \& Bournaud, F. 2010, MNRAS, 404, 2151 Chabrier, G. 2003, PASP, 115, 763

Cicone, C., Bothwell, M., Wagg, J., et al. 2017, A\&A, 604, A53 Circosta, C., Mainieri, V., Padovani, P., et al. 2018, A\&A, 620, A82 Civano, F., Marchesi, S., Comastri, A., et al. 2016, ApJ, 819, 62 Coogan, R. T., Daddi, E., Sargent, M. T., et al. 2018, MNRAS, 479, 703 Cortzen, I., Magdis, G. E., Valentino, F., et al. 2020, A\&A, 634, L14 Daddi, E., Dickinson, M., Morrison, G., et al. 2007, ApJ, 670, 156 Daddi, E., Bournaud, F., Walter, F., et al. 2010a, ApJ, 713, 686 Daddi, E., Elbaz, D., Walter, F., et al. 2010b, ApJ, 714, L118 Daddi, E., Dannerbauer, H., Liu, D., et al. 2015, A\&A, 577, A46 Dale, D. A., Aniano, G., Engelbracht, C. W., et al. 2012, ApJ, 745, 95 Dale, D. A., Cook, D. O., Roussel, H., et al. 2017, ApJ, 837, 90 Dannerbauer, H., Daddi, E., Riechers, D. A., et al. 2009, ApJ, 698, L178 Dannerbauer, H., Harrington, K., Díaz-Sánchez, A., et al. 2019, AJ, 158, 34 Decarli, R., Walter, F., Gónzalez-López, J., et al. 2019, ApJ, 882, 138 Dekel, A., \& Burkert, A. 2014, MNRAS, 438, 1870

Dekel, A., Birnboim, Y., Engel, G., et al. 2009a, Nature, 457, 451

Dekel, A., Sari, R., \& Ceverino, D. 2009b, ApJ, 703, 785

Donley, J. L., Koekemoer, A. M., Brusa, M., et al. 2012, ApJ, 748, 142 Draine, B. T., \& Li, A. 2007, ApJ, 657, 810

Drew, P. M., Casey, C. M., Cooray, A., \& Whitaker, K. E. 2020, ApJ, 892, 104 Dudzevičiūtè, U., Smail, I., Swinbank, A. M., et al. 2020, MNRAS, 494, 3828 Dunlop, J. S., McLure, R. J., Biggs, A. D., et al. 2017, MNRAS, 466, 861 Elbaz, D., Daddi, E., Le Borgne, D., et al. 2007, A\&A, 468, 33 Elbaz, D., Dickinson, M., Hwang, H. S., et al. 2011, A\&A, 533, A119 Elbaz, D., Leiton, R., Nagar, N., et al. 2018, A\&A, 616, A110

Elmegreen, D. M., Elmegreen, B. G., Ravindranath, S., \& Coe, D. A. 2007, ApJ, 658,763

Fensch, J., Renaud, F., Bournaud, F., et al. 2017, MNRAS, 465, 1934

Fixsen, D. J., Bennett, C. L., \& Mather, J. C. 1999, ApJ, 526, 207

Flower, D. R. 2001, J. Phys. B At. Mol. Phys., 34, 2731

Förster Schreiber, N. M., Shapley, A. E., Genzel, R., et al. 2011, ApJ, 739, 45

Franco, M., Elbaz, D., Béthermin, M., et al. 2018, A\&A, 620, A152

Freundlich, J., Combes, F., Tacconi, L. J., et al. 2019, A\&A, 622, A105

Gao, Y., Xiao, T., Li, C., et al. 2019, ApJ, 887, 172

Geach, J. E., Dunlop, J. S., Halpern, M., et al. 2017, MNRAS, 465, 1789

Genzel, R., Tacconi, L. J., Gracia-Carpio, J., et al. 2010, MNRAS, 407, 2091

Genzel, R., Newman, S., Jones, T., et al. 2011, ApJ, 733, 101

Genzel, R., Tacconi, L. J., Lutz, D., et al. 2015, ApJ, 800, 20

Glover, S. C. O., Clark, P. C., Micic, M., \& Molina, F. 2015, MNRAS, 448, 1607

Goldreich, P., \& Kwan, J. 1974, ApJ, 189, 441

Gómez-Guijarro, C., Toft, S., Karim, A., et al. 2018, ApJ, 856, 121

Gómez-Guijarro, C., Magdis, G. E., Valentino, F., et al. 2019, ApJ, 886, 88

Greve, T. R., Papadopoulos, P. P., Gao, Y., \& Radford, S. J. E. 2009, ApJ, 692, 1432

Greve, T. R., Leonidaki, I., Xilouris, E. M., et al. 2014, ApJ, 794, 142

Groves, B. A., Schinnerer, E., Leroy, A., et al. 2015, ApJ, 799, 96

Guilloteau, S., \& Lucas, R. 2000, in Imaging at Radio through Submillimeter Wavelengths, eds. J. G. Mangum, \& S. J. E. Radford, ASP Conf. Ser., 217, 299

Guo, Y., Giavalisco, M., Ferguson, H. C., Cassata, P., \& Koekemoer, A. M. 2012, ApJ, 757, 120

Guo, Y., Ferguson, H. C., Bell, E. F., et al. 2015, ApJ, 800, 39

Hoaglin, D. C., Mosteller, F., \& Tukey, J. W. 1983, Understanding Robust and Exploratory Data Analysis (New York: Wiley)

Hodge, J. A., \& da Cunha, E. 2020, ArXiv e-prints [arXiv:2004.00934]

Hodge, J. A., Swinbank, A. M., Simpson, J. M., et al. 2016, ApJ, 833, 103

Hodge, J. A., Smail, I., Walter, F., et al. 2019, ApJ, 876, 130

Huang, J. S., Rigopoulou, D., Magdis, G., et al. 2014, ApJ, 784, 52

Ivison, R. J., Papadopoulos, P. P., Smail, I., et al. 2011, MNRAS, 412, 1913

Jiménez-Andrade, E. F., Magnelli, B., Karim, A., et al. 2018, A\&A, 615, A25

Jiménez-Andrade, E. F., Magnelli, B., Karim, A., et al. 2019, A\&A, 625, A114

Jin, S., Daddi, E., Liu, D., et al. 2018, ApJ, 864, 56

Jin, S., Daddi, E., Magdis, G. E., et al. 2019, ApJ, 887, 144

Kamenetzky, J., Glenn, J., Rangwala, N., et al. 2012, ApJ, 753, 70

Kamenetzky, J., Rangwala, N., Glenn, J., Maloney, P. R., \& Conley, A. 2016, ApJ, 829, 93

Kamenetzky, J., Rangwala, N., \& Glenn, J. 2017, MNRAS, 471, 2917

Kaplan, E. L., \& Meier, P. 1958, J. Am. Stat. Assoc., 53, 457

Kelly, B. C. 2007, ApJ, 665, 1489

Kennicutt, R. C., Jr 1998, ApJ, 498, 541

Kennicutt, R. C., Calzetti, D., Aniano, G., et al. 2011, PASP, 123, 1347 
Kereš, D., Katz, N., Weinberg, D. H., \& Davé, R. 2005, MNRAS, 363, 2 Laigle, C., McCracken, H. J., Ilbert, O., et al. 2016, ApJS, 224, 24

Lee, M. Y., Madden, S. C., Le Petit, F., et al. 2019, A\&A, 628, A113

Le Fèvre, O., Béthermin, M., \& Faisst, A. 2020, A\&A, in press, https: //doi. org/10.1051/0004-6361/201936965

Leroy, A. K., Walter, F., Brinks, E., et al. 2008, AJ, 136, 2782

Leroy, A. K., Walter, F., Bigiel, F., et al. 2009, AJ, 137, 4670

Li, A., \& Draine, B. T. 2001, ApJ, 554, 778

Liu, D. 2020, Michi2: SED and SLED Fitting Tool

Liu, D., Gao, Y., Isaak, K., et al. 2015a, ApJ, 810, L14

Liu, L., Gao, Y., \& Greve, T. R. 2015b, ApJ, 805, 31

Liu, D., Daddi, E., Dickinson, M., et al. 2018, ApJ, 853, 172

Liu, D., Schinnerer, E., Groves, B., et al. 2019a, ApJ, 887, 235

Liu, D., Lang, P., Magnelli, B., et al. 2019b, ApJS, 244, 40

Lu, N., Zhao, Y., Xu, C. K., et al. 2014, ApJ, 787, L23

Lu, N., Zhao, Y., Xu, C. K., et al. 2015, ApJ, 802, L11

Lu, N., Zhao, Y., Díaz-Santos, T., et al. 2017, ApJS, 230, 1

Lutz, D., Poglitsch, A., Altieri, B., et al. 2011, A\&A, 532, A90

Lutz, D., Berta, S., Contursi, A., et al. 2016, A\&A, 591, A136

Magdis, G. E., Daddi, E., Béthermin, M., et al. 2012, ApJ, 760, 6

Magdis, G. E., Rigopoulou, D., Daddi, E., et al. 2017, A\&A, 603, A93

Mannucci, F., Cresci, G., Maiolino, R., Marconi, A., \& Gnerucci, A. 2010 MNRAS, 408, 2115

Marchesi, S., Civano, F., Elvis, M., et al. 2016, ApJ, 817, 34

Mashian, N., Sturm, E., Sternberg, A., et al. 2015, ApJ, 802, 81

McMullin, J. P., Waters, B., Schiebel, D., Young, W., \& Golap, K. 2007, in Astronomical Data Analysis Software and Systems XVI, eds. R. A. Shaw, F. Hill, \& D. J. Bell, ASP Conf. Ser., 376, 127

Mullaney, J. R., Alexander, D. M., Goulding, A. D., \& Hickox, R. C. 2011, MNRAS, 414, 1082

Muzzin, A., Wilson, G., Demarco, R., et al. 2013, ApJ, 767, 39

Narayanan, D., \& Krumholz, M. R. 2014, MNRAS, 442, 1411

Noeske, K. G., Weiner, B. J., Faber, S. M., et al. 2007, ApJ, 660, L43

Noll, S., Burgarella, D., Giovannoli, E., et al. 2009, A\&A, 507, 1793

Oliver, S. J., Bock, J., Altieri, B., et al. 2012, MNRAS, 424, 1614

Pan, H.-A., Lin, L., Hsieh, B.-C., et al. 2018, ApJ, 868, 132

Panuzzo, P., Rangwala, N., Rykala, A., et al. 2010, A\&A, 518, L37

Papadopoulos, P. P., \& Seaquist, E. R. 1998, ApJ, 492, 521

Papadopoulos, P. P., Thi, W.-F., \& Viti, S. 2004, MNRAS, 351, 147

Papadopoulos, P. P., Isaak, K., \& van der Werf, P. 2010a, ApJ, 711, 757

Papadopoulos, P. P., van der Werf, P., Isaak, K., \& Xilouris, E. M. 2010b, ApJ, 715,775

Papadopoulos, P. P., van der Werf, P. P., Xilouris, E. M., et al. 2012, MNRAS, 426, 2601

Popping, G., Decarli, R., Man, A. W. S., et al. 2017, A\&A, 602, A11

Puglisi, A., Daddi, E., Renzini, A., et al. 2017, ApJ, 838, L18

Puglisi, A., Daddi, E., Liu, D., et al. 2019, ApJ, 877, L23

Rangwala, N., Maloney, P. R., Glenn, J., et al. 2011, ApJ, 743, 94

Riechers, D. A., Bradford, C. M., Clements, D. L., et al. 2013, Nature, 496, 329

Riechers, D. A., Pavesi, R., Sharon, C. E., et al. 2019, ApJ, 872, 7

Rigopoulou, D., Hurley, P. D., Swinyard, B. M., et al. 2013, MNRAS, 434, 2051

Rodighiero, G., Daddi, E., Baronchelli, I., et al. 2011, ApJ, 739, L40

Rosenberg, M. J. F., van der Werf, P. P., Aalto, S., et al. 2015, ApJ, 801, 72

Rujopakarn, W., Rieke, G. H., Eisenstein, D. J., \& Juneau, S. 2011, ApJ , 726, 93
Saintonge, A., Catinella, B., Tacconi, L. J., et al. 2017, ApJS, 233, 22 Sanders, D. B., \& Mirabel, I. F. 1996, ARA\&A, 34, 749

Sanders, D. B., Mazzarella, J. M., Kim, D.-C., Surace, J. A., \& Soifer, B. T. 2003, AJ, 126, 1607

Sanders, D. B., Salvato, M., Aussel, H., et al. 2007, ApJS, 172, 86 Sargent, M. T., Daddi, E., Béthermin, M., et al. 2014, ApJ, 793, 19 Schinnerer, E., Sargent, M. T., Bondi, M., et al. 2010, ApJS, 188, 384 Schirm, M. R. P., Wilson, C. D., Parkin, T. J., et al. 2014, ApJ, 781, 101 Schmidt, M. 1959, ApJ, 129, 243

Schreiber, C., Pannella, M., Elbaz, D., et al. 2015, A\&A, 575, A74

Schreiber, C., Elbaz, D., Pannella, M., et al. 2018, A\&A, 609, A30 Scoville, N. Z., \& Solomon, P. M. 1974, ApJ, 187, L67

Scoville, N., Aussel, H., Brusa, M., et al. 2007, ApJS, 172, 1

Scoville, N., Sheth, K., Aussel, H., et al. 2016, ApJ, 820, 83

Scoville, N., Lee, N., Vanden Bout, P., et al. 2017a, ApJ, 837, 150

Scoville, N., Murchikova, L., Walter, F., et al. 2017b, ApJ, 836, 66

Silverman, J. D., Daddi, E., Rodighiero, G., et al. 2015, ApJ, 812, L23

Silverman, J. D., Daddi, E., Rujopakarn, W., et al. 2018a, ApJ, 868, 75

Silverman, J. D., Rujopakarn, W., Daddi, E., et al. 2018b, ApJ, 867, 92

Simpson, J. M., Swinbank, A. M., Smail, I., et al. 2014, ApJ, 788, 125

Simpson, J. M., Smail, I., Wang, W.-H., et al. 2017, ApJ, 844, L10

Simpson, J. M., Smail, I., Dudzeviciute, U., et al. 2020, MNRAS, 495, 3409

Smail, I., Ivison, R. J., \& Blain, A. W. 1997, ApJ, 490, L5

Smolčić, V., Novak, M., Bondi, M., et al. 2017, A\&A, 602, A1

Solomon, P. M., \& Vanden Bout, P. A. 2005, ARA\&A, 43, 677

Speagle, J. S., Steinhardt, C. L., Capak, P. L., \& Silverman, J. D. 2014, ApJS, 214,15

Spilker, J. S., Marrone, D. P., Aguirre, J. E., et al. 2014, ApJ, 785, 149

Spilker, J. S., Marrone, D. P., Aravena, M., et al. 2016, ApJ, 826, 112

Strandet, M. L., Weiss, A., De Breuck, C., et al. 2017, ApJ, 842, L15

Tacchella, S., Dekel, A., Carollo, C. M., et al. 2016, MNRAS, 457, 2790

Tacconi, L. J., Genzel, R., Neri, R., et al. 2010, Nature, 463, 781

Tacconi, L. J., Genzel, R., Saintonge, A., et al. 2018, ApJ, 853, 179

Tacconi, L. J., Genzel, R., \& Sternberg, A. 2020, ARA\&A, submitted [arXiv:2003.06245]

Tress, R. G., Smith, R. J., Sormani, M. C., et al. 2020, MNRAS, 492, 2973

Valentino, F., Magdis, G. E., Daddi, E., et al. 2018, ApJ, 869, 27

Valentino, F., Magdis, G. E., Daddi, E., et al. 2020, ApJ, 890, 24

van der Tak, F. F. S., Black, J. H., Schöier, F. L., Jansen, D. J., \& van Dishoeck, E. F. 2007 , A\&A, 468, 627

Vollmer, B., Gratier, P., Braine, J., \& Bot, C. 2017, A\&A, 602, A51

Walter, F., Weiß, A., Downes, D., Decarli, R., \& Henkel, C. 2011, ApJ, 730, 18

Weiß, A., Henkel, C., Downes, D., \& Walter, F. 2003, A\&A, 409, L41

Weiß, A., Walter, F., \& Scoville, N. Z. 2005, A\&A, 438, 533

Weiß, A., Downes, D., Neri, R., et al. 2007, A\&A, 467, 955

Whitaker, K. E., van Dokkum, P. G., Brammer, G., \& Franx, M. 2012, ApJ, 754, L29

Whitaker, K. E., Franx, M., Leja, J., et al. 2014, ApJ, 795, 104

Wu, R., Madden, S. C., Galliano, F., et al. 2015, A\&A, 575, A88

Yang, C., Omont, A., Beelen, A., et al. 2017, A\&A, 608, A144

Young, J. S., \& Scoville, N. Z. 1991, ARA\&A, 29, 581

Zanella, A., Daddi, E., Le Floc'h, E., et al. 2015, Nature, 521, 54

Zanella, A., Daddi, E., Magdis, G., et al. 2018, MNRAS, 481, 1976

Zanella, A., Le Floc'h, E., Harrison, C. M., et al. 2019, MNRAS, 489, 2792

Zavala, J. A., Casey, C. M., da Cunha, E., et al. 2018, ApJ, 869, 71 


\section{Appendix A: Total recovered fluxes}

The total flux of a source can be robustly recovered if its size is securely estimated. However, our iterative extraction could drive to flux losses when we estimate only an upper limit on the size, and such value is comparable with the beam size. We estimated these losses as detailed in Appendix B of V20, namely by injecting artificial bright galaxies with circular Gaussian profiles and a FWHM fixed to the $1 \sigma$ upper limit on the size in the $u v$ plane, and then re-extracting their fluxes with the fiducial point source profile. We then corrected the extracted fluxes to $\left(I_{\text {Gauss }} / I_{\text {Point }}+1\right) / 2$ and added in quadrature the absolute error on such correction $\left(\sigma_{\text {corr }}=\left(I_{\text {Gauss }}-I_{\text {Point }}\right) / 2\right)$ to the statistical uncertainty. The correction does not depend on the brightness of the injected mock source, provided that it is significantly detected $(S / N \geq 10)$, nor on its position in the map. The sizes and the flux corrections are reported in the data release.

\section{Appendix B: Revisiting the target selection a posteriori}

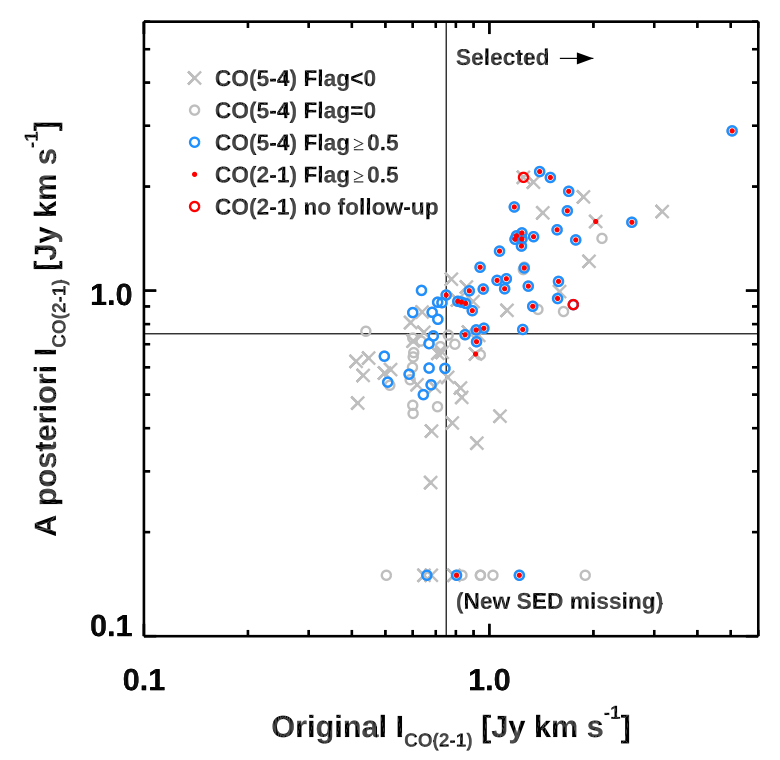

Fig. B.1. Revisiting the selection and the impact on the detection rate. Predicted $I_{21}$ fluxes with the original $L_{\mathrm{IR}}$ based on PACS detections and on the new IR photometry from Jin et al. (2018). The symbols mark the whole parent sample of 123 galaxies with ALMA Band 6 observations targeting $\mathrm{CO}(5-4)$. Gray crosses, gray open circles, and blue open circles indicate sources with uncertain and reliable information on $\mathrm{CO}(5-4)$ (Flag $<0,0, \geq 0.5$, respectively, Sect. 3.2.2). Red filled circles show galaxies with reliable information on $\mathrm{CO}(2-1)$ (Flag $\geq 0.5)$. Open red circles mark objects with predicted fluxes bright enough to be observed according to both the initial and the revised SED modeling, but that did not enter the final selection due to the frequency grouping. The black solid lines indicate the depth of the ALMA Band 3 observations: objects on the right side of the vertical line were selected for the follow-up (excluding the red open circles); object above the horizontal line could have been selected, if the updated photometry were available when preparing the observations. Objects with missing photometry in Jin et al. (2018) are artificially set to $I_{\mathrm{CO}(2-1)}=0.15 \mathrm{Jy} \mathrm{km} \mathrm{s}^{-1}$ and labeled accordingly.

Reconstructing a posteriori the target selection for the $\mathrm{CO}(2-1)$ follow-up observations, with the improved constraints on the IR photometry that became available in the meantime, allows us to get a handle on the factors determining the detection rates described in Sect. 3.2.2. In Fig. B.1, we show the original prediction of the $I_{\mathrm{CO}(2-1)}^{\mathrm{pred}}$ fluxes computed from previous $L_{\mathrm{IR}}$ estimates from the PEP survey (Sect. 2), against an updated version based on the far-IR modeling of the deblended photometry from Jin et al. (2018). Excluding 12 sources from the PEP survey without a counterpart in the deblended catalog, the flux predictions scatter around the one-to-one relation. Galaxies in the bottom right quadrant of Fig. B.1 were bright enough to be selected for the follow-up observations, but they would have missed the cut based on the updated $L_{\mathrm{IR}}$. The negligible fraction of $\mathrm{CO}(2-1)$ detections among these objects supports the hypothesis that they are indeed too faint to be detected at the current depth. On the other hand, for the sources in the top right quadrant of Fig. B. 1 the new photometric modeling supports the initial selection. In fact, this is where virtually all $\mathrm{CO}(2-1)$ detections are located. Undetected objects primarily lack reliable information of $\mathrm{CO}(5-4)$ and have low quality flags on the optical or nearIR spectroscopic redshifts $z_{\text {spec,opt }}$. Only a minor fraction of IRbright sources do have secure $z_{\text {spec,opt }}$, but remained undetected in $\mathrm{CO}(5-4)$ and $\mathrm{CO}(2-1)$, likely due to bona fide dimmer line fluxes than predicted. Galaxies in the remaining left quadrants were not selected for the $\mathrm{CO}(2-1)$ follow-up and their $\mathrm{CO}(5-4)$ detection rate is set by a combination of bad $z_{\text {spec,opt }}$, lower $L_{\mathrm{IR}}$ than previously estimated, and intrinsically faint lines in bright objects, in order of importance. The latter are physically interesting, but we cannot currently put any constraints on their properties, in absence of a secure $z_{\text {spec,submm }}$.

\section{Appendix C: The diversity of the CO SLEDs of galaxies on and above the main sequence}

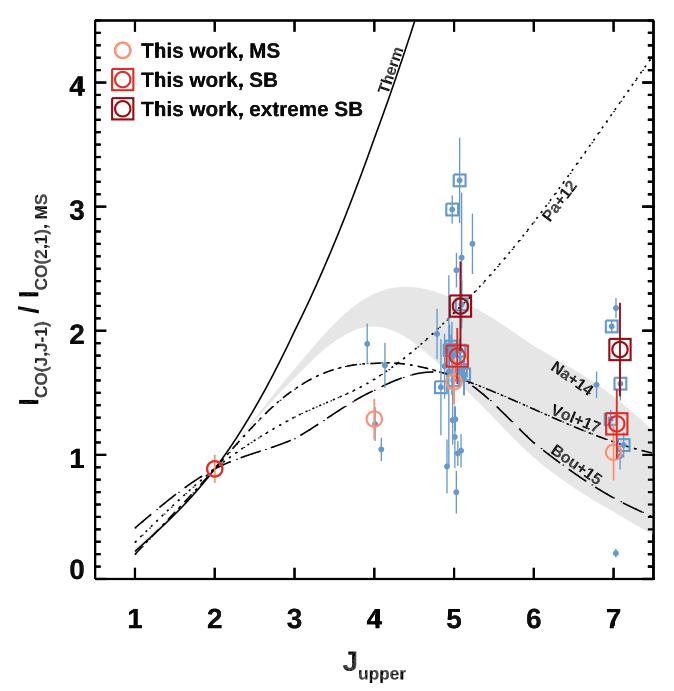

Fig. C.1. Diversity of CO SLEDs for main-sequence and starburst galaxies. Blue filled circles and open squares indicate the mainsequence and starburst galaxies with detected $\mathrm{CO}(5-4)$ and $\mathrm{CO}(2-1)$ lines from our ALMA follow-up, normalized to the mean CO $(2-1)$ flux for the main-sequence sample. The open red circles and squares indicate the mean fluxes for main-sequence, starburst ( $\triangle \mathrm{MS} \geq 3.5$ ), and extreme starburst $(\Delta \mathrm{MS} \geq 7)$ objects. The solid line shows the line ratios for a fully thermalized case. The gray shaded area marks the model by Narayanan \& Krumholz (2014) for unresolved observations within $\Sigma_{\mathrm{SFR}}=1-10 M_{\odot} \mathrm{yr}^{-1} \mathrm{kpc}^{-2}$. The long dashed line indicates the simulations from Bournaud et al. (2015). The dotted line traces the empirical model by Papadopoulos et al. (2012). The dashed-dotted line points at the analytical model by Vollmer et al. (2017). 
In Fig. C.1 we show the CO SLEDs for the subsample of main-sequence and starburst galaxies with $\mathrm{CO}(2-1)$ and $\mathrm{CO}(5-4)$ detections from our ALMA observations, normalized to the mean $\mathrm{CO}(2-1)$ flux for the main-sequence sample (Sect. 4.4). This figure highlights the variety of shapes displayed even by a homogeneously selected sample of normal and extreme galaxies at high redshift.

\section{Appendix D: Data tables, galaxy spectra, and spectral energy distribution}

Figure 2 shows an example of the ALMA spectra and the IR SED for our sample of reliable sources used in the analysis (Flag $\geq 0.5$ ). The whole compilation of spectra from which we extracted reliable information is available online ${ }^{5}$. Similarly, the full data table is made public in electronic format format. The description of the columns is listed in Table D.1.

Table D.1. Column description for the data release.

\begin{tabular}{|c|c|c|}
\hline Name & Units & Description \\
\hline ID & & Identifier \\
\hline RA & hh:mm:ss & Right ascension \\
\hline Dec & dd:mm:ss & Declination \\
\hline zspec_opticalnir & $\ldots$ & Optical or near-IR spectroscopic redshift (Salvato et al., in prep.) \\
\hline (d)zspec_submm & $\ldots$ & ALMA submillimeter spectroscopic redshift \\
\hline Log_StellarMass & $M_{\odot}$ & Logarithm of the stellar mass (Chabrier 2003 IMF) \\
\hline (d)Total_LIR & $L_{\odot}$ & Total 8-1000 $\mu \mathrm{m} L_{\mathrm{IR}}$ (Draine \& Li 2007; Mullaney et al. 2011) \\
\hline (d)SF_LIR & $L_{\odot}$ & $L_{\mathrm{IR}, \mathrm{SFR}}$ from the star-forming component $\left(L_{\mathrm{IR}, \mathrm{SFR}}=L_{\mathrm{IR}}-L_{\mathrm{IR}, \mathrm{AGN}}\right)$ \\
\hline (d)AGN_LIR & $L_{\odot}$ & $L_{\mathrm{IR}, \mathrm{AGN}}$ from the AGN component $\left(L_{\mathrm{IR}, \mathrm{AGN}}=L_{\mathrm{IR}} \times f_{\mathrm{AGN}}\right)$ \\
\hline (d) $f_{\mathrm{AGN}}$ & $\ldots$ & Fraction of $L_{\mathrm{IR}}$ due to the AGN emission \\
\hline (d) $M_{\text {dust }}$ & $M_{\odot}$ & Dust mass (Draine \& Li 2007) \\
\hline (d) $U$ & $\ldots$ & Mean intensity of the interstellar radiation field (Draine \& Li 2007) \\
\hline DistanceMS & $\ldots$ & Distance from the main sequence as parameterized in Sargent et al. (2014) \\
\hline (d)Size & $\operatorname{arcsec}$ & Source angular size from ALMA \\
\hline OneSigma_Size & $\operatorname{arcsec}$ & $1 \sigma$ upper limit on the source angular size from ALMA \\
\hline Probability_Unresolved & & Probability of being unresolved (Puglisi et al. 2019) \\
\hline Flux_Line $(X)$ & $\mathrm{Jy} \mathrm{km} \mathrm{s}^{-1}$ & Velocity integrated flux of line $X$ \\
\hline SNR_ $(X)$ & $\ldots$ & Signal to noise ratio of the flux of line $X$ \\
\hline OneSigma_ $(X)$ & $\mathrm{Jy} \mathrm{km} \mathrm{s}^{-1}$ & $1 \sigma$ upper limit on the flux of line $X$ \\
\hline Width_(X) & $\mathrm{km} \mathrm{s}^{-1}$ & Velocity width of line $X$ \\
\hline (d)ApertureCorr_(X) & $\ldots$ & Applied aperture correction for line $X$ \\
\hline Prob_Line $(X)$ & $\ldots$ & Probability of spurious detection of line $X$ \\
\hline Flag_(X) & $\ldots$ & Quality and usage flag for line $X$ \\
\hline $\begin{array}{l}\text { FreqContinuumBand }(B) \_(X) \\
\text { (d)ContinuumBand }(B) \_(X)\end{array}$ & $\begin{array}{l}\mathrm{GHz} \\
\mathrm{mJy}\end{array}$ & $\begin{array}{c}\text { Frequency in Band } B \text { under line } X \text { for the estimate of the continuum emission } \\
\text { Continuum emission in Band } B \text { under line } X\end{array}$ \\
\hline
\end{tabular}

Notes. This full table is available at the CDS. Lines $X$ : $\mathrm{CO}(5-4), \mathrm{CO}(2-1), \mathrm{CO}(7-6),[\mathrm{C} \mathrm{I}]\left({ }^{3} P_{2}-{ }^{3} P_{1}\right),[\mathrm{C} \mathrm{I}]\left({ }^{3} P_{1}-{ }^{3} P_{0}\right)$, and $\mathrm{CO}(4-3)$. Bands $B$ : Band 6, Band 3, and Band 7. The uncertainties have the same name of the quantity that they refer to, preceded by $d$ (e.g., Total_LIR \pm dTotal_LIR). 